\title{
Tetraspanin TM4SF5 mediates loss of contact inhibition through epithelial-mesenchymal transition in human hepatocarcinoma
}

\begin{abstract}
Sin-Ae Lee,1,2 Sung-Yul Lee, ${ }^{1,2}$ Ik-Hyun Cho, ${ }^{3}$ Min-A Oh, ${ }^{1,4}$ Eun-Sil Kang,, ${ }^{1,2}$ Yong-Bae Kim, ${ }^{1,4}$ Woo Duck Seo, ${ }^{5}$ Suyong Choi, ${ }^{1,4}$ Ju-Ock Nam, ${ }^{6}$ Mimi Tamamori-Adachi, ${ }^{7}$ Shigetaka Kitajima, ${ }^{7}$ Sang-Kyu Ye, ${ }^{8}$ Semi Kim, ${ }^{9}$ Yoon-Jin Hwang, ${ }^{10}$ In-San Kim, ${ }^{6}$ Ki Hun Park, ${ }^{5}$ and Jung Weon Lee ${ }^{1,2,4}$

${ }^{1}$ Cancer Research Institute, College of Medicine, and Cell Dynamics Research Center, ${ }^{2}$ Department of Molecular and Clinical Oncology, ${ }^{3}$ College of Dentistry and Dental Research Institute, and ${ }^{4}$ Department of Tumor Biology, Seoul National University, Seoul, Republic of Korea. ${ }^{5}$ Division of Applied Life Science, Gyeongsang National University, Jinju, Republic of Korea. ${ }^{6}$ Department of Biochemistry, Kyungpook National University School of Medicine, Daegu, Republic of Korea. ${ }^{7}$ Department of Biochemical Genetics, Tokyo Medical and Dental University, Tokyo, Japan. ${ }^{8}$ Department of Pharmacology, College of Medicine, Seoul National University, Seoul, Republic of Korea. ${ }^{9}$ Korea Research Institute of Bioscience and Biotechnology, Daejeon, Republic of Korea. ${ }^{10}$ Department of Surgery, Kyungpook National University School of Medicine, Daegu, Republic of Korea.
\end{abstract}

\begin{abstract}
The growth of normal cells is arrested when they come in contact with each other, a process known as contact inhibition. Contact inhibition is lost during tumorigenesis, resulting in uncontrolled cell growth. Here, we investigated the role of the tetraspanin transmembrane 4 superfamily member 5 (TM4SF5) in contact inhibition and tumorigenesis. We found that TM4SF5 was overexpressed in human hepatocarcinoma tissue. TM4SF5 expression in clinical samples and in human hepatocellular carcinoma cell lines correlated with enhanced $\mathrm{p} 27^{\mathrm{Kip} 1}$ expression and cytosolic stabilization as well as morphological elongation mediated by RhoA inactivation. These TM4SF5-mediated effects resulted in epithelial-mesenchymal transition (EMT) via loss of E-cadherin expression. The consequence of this was aberrant cell growth, as assessed by $S$-phase transition in confluent conditions, anchorage-independent growth, and tumor formation in nude mice. The TM4SF5mediated effects were abolished by suppressing the expression of either TM4SF 5 or cytosolic P27Kip1, as well as by reconstituting the expression of E-cadherin. Our observations have revealed a role for TM4SF5 in causing uncontrolled growth of human hepatocarcinoma cells through EMT.
\end{abstract}

\section{Introduction}

Epithelial monolayer organization involves cell adhesion via integrin engagement to ECM proteins within basement membranes and cell-cell contacts between adjacent cells through homophilic interactions of adhesion molecules, including E-cadherin and others (1). Since adhesion receptors at focal adhesion and cell-cell contact sites are linked to actin filaments through protein complexes at their cytoplasmic tails, reorganization of actin filaments can cause loss of focal adhesions and cell-cell contacts, leading to an epithelial-mesenchymal transition (EMT) that consequently disrupts monolayer integrity (2).

Transmembrane 4 superfamily (TM4SF) proteins (also known as tetraspanins or tetraspans) are a group of hydrophobic proteins of approximately $25-50 \mathrm{kDa}$ with 4 transmembrane domains, 2 extracellular loops, and 2 short cytoplasmic tails (3). TM4SFs form massive tetraspanin web structures by forming complexes with cell adhesion molecules, such as integrins, to collaboratively perform their roles in cell adhesion, proliferation, and motility (4-6). Integrins, a group of cell adhesion receptors, activate diverse intracellular signaling molecules and reorganize actin filaments (7-9). Signaling molecules activated by integrin include focal

Nonstandard abbreviations used: EMT, epithelial-mesenchymal transition; FAK, focal adhesion kinase; GAP, GTPase-activating protein; GRAF, GTPase regulator associated with FAK; HGF, hepatocyte growth factor; KIS, kinase interacting with stathmin; LPA, lysophosphatidic acid; TM4SF5, transmembrane 4 superfamily member 5 . Conflict of interest: The authors have declared that no conflict of interest exists. Citation for this article: J. Clin. Invest. 118:1354-1366 (2008). doi:10.1172/JCI33768. adhesion kinase (FAK), RhoA GTPases, and others $(10,11)$. RhoA GTPases consisting of RhoA, Rac1, and CDC42 critically regulate actin reorganization (12) through their downstream effector molecules, including LIMK, PAK1, MLCK, mDia, or ROCK, to affect actin polymerization and myosin light chain phosphorylationmediated intracellular contractility (13). Recently, $\mathrm{p} 27^{\mathrm{Kip} 1}$, a cyclindependent kinase (CDK) inhibitor, was shown to inhibit the RhoA pathway via direct interaction in the cytosol (14). The cytosolic localization and stabilization of $\mathrm{p} 27^{\mathrm{Kip} 1}$ were dependent on its Ser10 phosphorylation by kinase interacting with stathmin (KIS; ref. 15) and PKB/Akt (16), and cytosolic stabilization of p27 Kip1 was shown in such diverse tumors as colon carcinoma, thyroid tumor, and Barrett's-associated adenocarcinoma (17-19).

TM4SF5 is a homolog of tumor-associated antigen L6 (TM4SF1) and forms a 4-transmembrane L6 superfamily with L6, IL-TMP, and L6D (20). mRNA for TM4SF5 is highly expressed in pancreatic, gastric, colon, papilla vateri carcinoma and soft tissue sarcoma (21) and nonendocrine lung and ACTH (corticotropin)-negative bronchial carcinoid tumors (22). However, its potential oncogenic functions have not yet been explored. Recently, we reported that ectopic TM4SF5 expression in Cos7 cells resulted in actin reorganization and focal adhesion turnover, depending positively on $\alpha_{2}$ integrin and negatively on serum treatment (23).

In the current study, we found that TM4SF5 is overexpressed in hepatocarcinoma tissues, and we mechanistically explored how TM4SF5 mediates tumorigenicity in hepatocyte and nude mouse systems. We observed that TM4SF5 caused actin reorganization and EMT via increased p27 ${ }^{\mathrm{Kip} 1}$ expression/cytosolic stabilization 
A
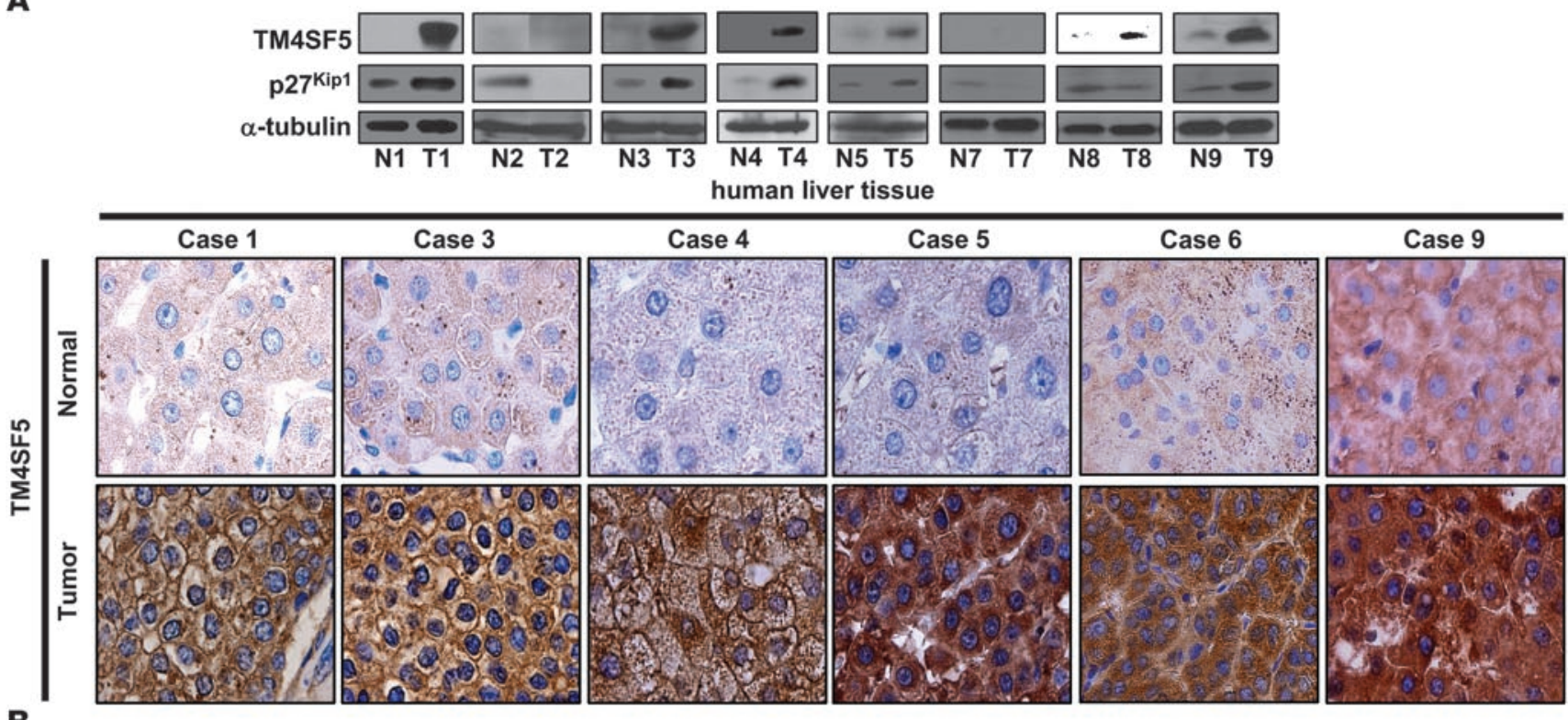

$\mathbf{B}$
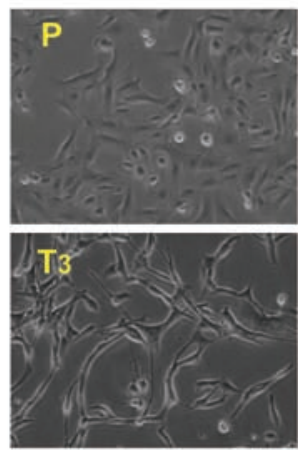
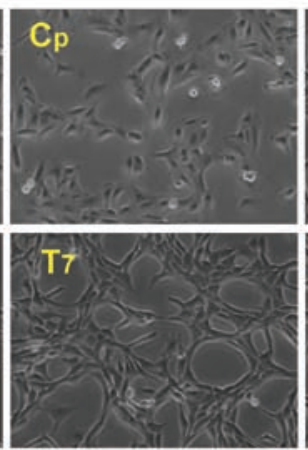
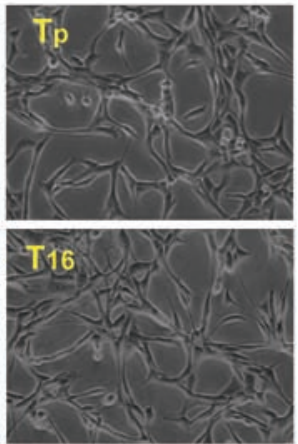

\section{TM4SF5}

$\alpha$-tubulin

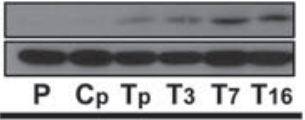

SNU449

\section{Figure 1}

TM4SF5 overexpression in hepatocarcinoma tissues and SNU449 hepatocyte model system. (A) TM4SF5 overexpression in hepatocarcinoma. Normal (N1-N9) and tumor (T1- T9) tissues separately obtained from 9 hepatocarcinoma patients were analyzed by standard Western blots (upper panel) and by immunohistochemistry (lower panel). N6 and T6 were prepared only for immunocytochemistry, because the dissected tissue was too small. Six representative pair cases are shown for TM4SF5 immunohistochemistry. (B) SNU449 cells without or with TM4SF5. Parental $(P)$ and stable SNU449 cells without $(\mathrm{Cp})$ or with TM4SF5 expression (Tp, T3, T7, and T16) were imaged and immunoblotted. Original magnification, $\times 400(\mathbf{A}) ; \times 100(B)$.

and decreased RhoA activity, leading to contact inhibition loss and oncogenic proliferation.

\section{Results}

TM4SF5-mediated RhoA inactivation and morphological elongation. Since TM4SF5 expression was shown to be higher in certain tumor types via Northern blot or RT-PCR analysis $(21,22)$, we examined whether it is overexpressed in hepatocarcinoma tissues compared with normal liver tissues. Seven of 9 tissues separately prepared from 9 hepatocarcinoma patients showed overexpression of TM4SF5 protein, compared with normal liver tissues; cases 2 and 7 did not express TM4SF5, and the tissue sample of case 6 was too small to perform immunoblots (Figure 1A). To study the oncogenic roles of TM4SF5, we established stable SNU449 hepatocytes ectopically expressing TM4SF5. Different single cell-driven clones (T3, T7, and T16) and a pooled clone (Tp) expressing TM4SF5 displayed elongated morphology, compared with TM4SF5-null parental SNU449 and control pooled (Cp) cells (Figure 1B). Actin stain- ing revealed that $\mathrm{Cp}$ cells had well-defined stress fibers supporting a widespread polygonal morphology, whereas Tp cells showed aberrant actin bundling along with an extension of cellular length (Supplemental Figure 1; supplemental material available online with this article; doi:10.1172/JCI33768DS1).

Since TM4SF5 was shown to be cooperative with integrinmediated signaling (23) and cellular morphology is controlled by integrin-mediated cell adhesion (24), the molecular basis for the TM4SF5-mediated morphological elongation was explored with regard to integrin signaling. Among molecules we screened, phosphorylations of FAK Tyr577 (i.e., pY ${ }^{577}$ FAK) and of c-Src Tyr416 ( $\mathrm{pY}^{416} \mathrm{c}-\mathrm{Src}$ ) were increased in TM4SF5-expressing cell clones, whereas RhoA activity was decreased (Figure 2, A and C). The TM4SF5-mediated increase in $\mathrm{PY}^{577} \mathrm{FAK}$ and decrease in RhoA activity were reversed by TM4SF5 knockdown via transfection with an shRNA targeting TM4SF5 (shTM4SF5), but not with control shRNA targeting GFP, or scrambled shRNA (Figure 2, B and $\mathrm{D}$, respectively). The shTM4SF5-transfected cells recovered 
A

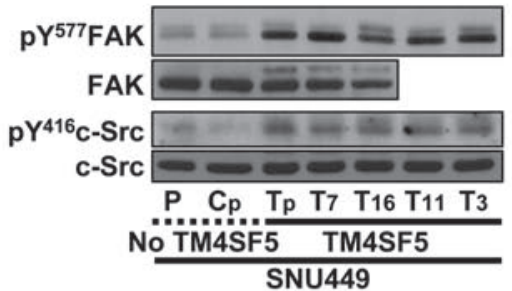

C

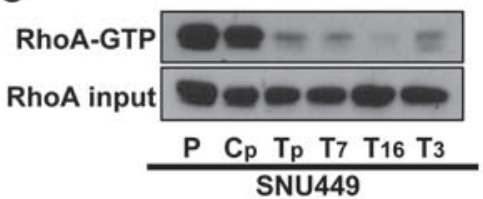

E

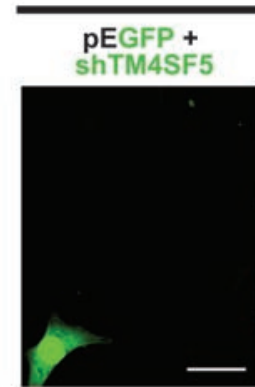

H

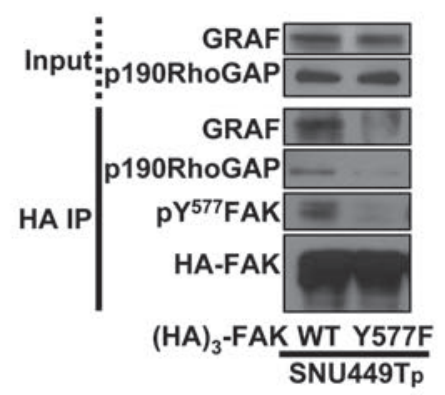

B

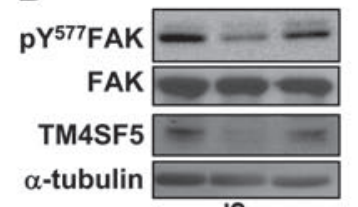

ShRNA 茪 $\sum_{\frac{1}{5}}^{\frac{\text { }}{5}}$

D

SNU449T16
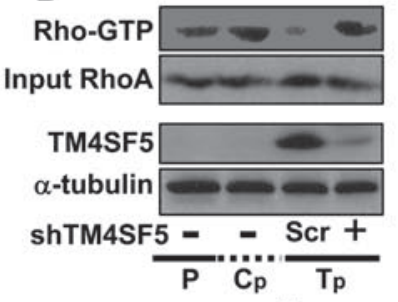

$\mathbf{F}$
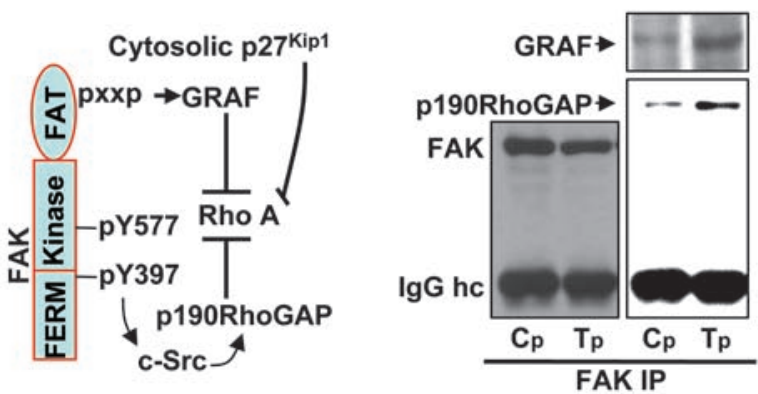

SNU449C $p$

I IshTM4SF5

J
Actin

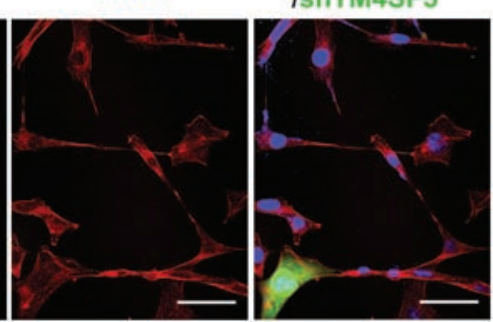

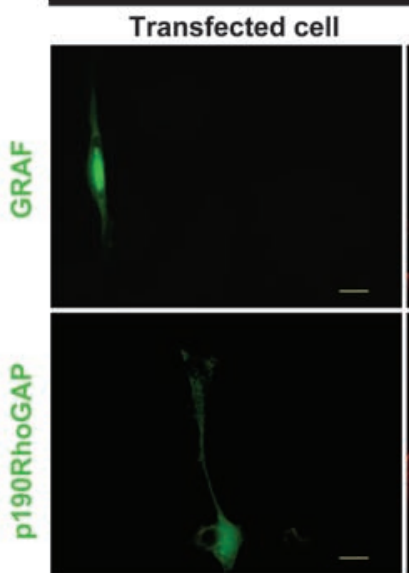
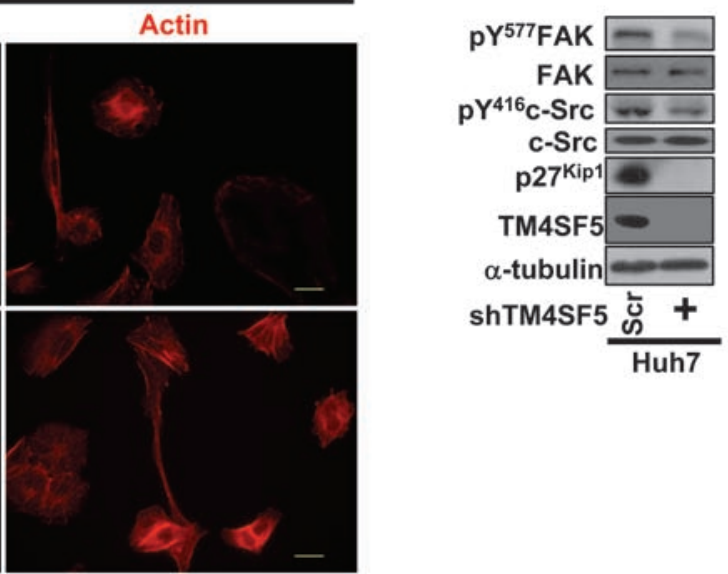

Figure 2

TM4SF5-mediated morphological elongation involves RhoA inactivation. Whole-cell lysates were immunoblotted (A) or used for RhoA assay and immunoblots (C). Parental, SNU449Cp, or TM4SF5-expressing cells transfected with shRNA against GFP, scrambled sequence (Scr), or TM4SF5 (shTM4SF5) were harvested for immunoblots (B) or RhoA activity assay and immunoblots (D). (E) SNU449Tp cells were cotransfected with pEGFP and shTM4SF5. After 24 hours, cells were replated on 10\% FBS/RPMI-1640-precoated coverslips. After an additional 24 hours, cells were stained for actin and DNA and analyzed by confocal microscopy. Scale bars: $20 \mu \mathrm{m}$. (F) Scheme showing the linkage from FAK to RhoA through RhoGAPs. FERM, FAK N-terminal band 4.1, ezrin, radixin, moesin homology domain; pxxp, proline-rich domain. (G) Increased association of RhoGAPs with FAK in SNU449Tp cells. Lysates from control (Cp) or SNU449Tp (Tp) cells were immunoprecipitated with anti-FAK antibody before immunoblot analysis. hc, heavy chain. (H) SNU449Tp cells were transiently transfected with (HA) ${ }_{3}-\mathrm{FAK}$ WT or Y577F mutant. After 48 hours, lysates were prepared and immunoprecipitated with anti-HA antibody, before immunoblot analysis. (I) SNU449Cp cells were transfected with pEGFP-FLAG-GRAF or p190RhoGAP. After 24 hours, cells were replated on coverslips, as above. After an additional 24 hours, cells were double-stained for actin and then for FLAG-tag or GFP. Scale bars: $10 \mu \mathrm{m}$. (J) Cell extracts from Huh7 cells stably transfected with scrambled shRNA or shTM4SF5 were used in immunoblots. Data shown represent 3 different experiments. 
A

human liver tissue
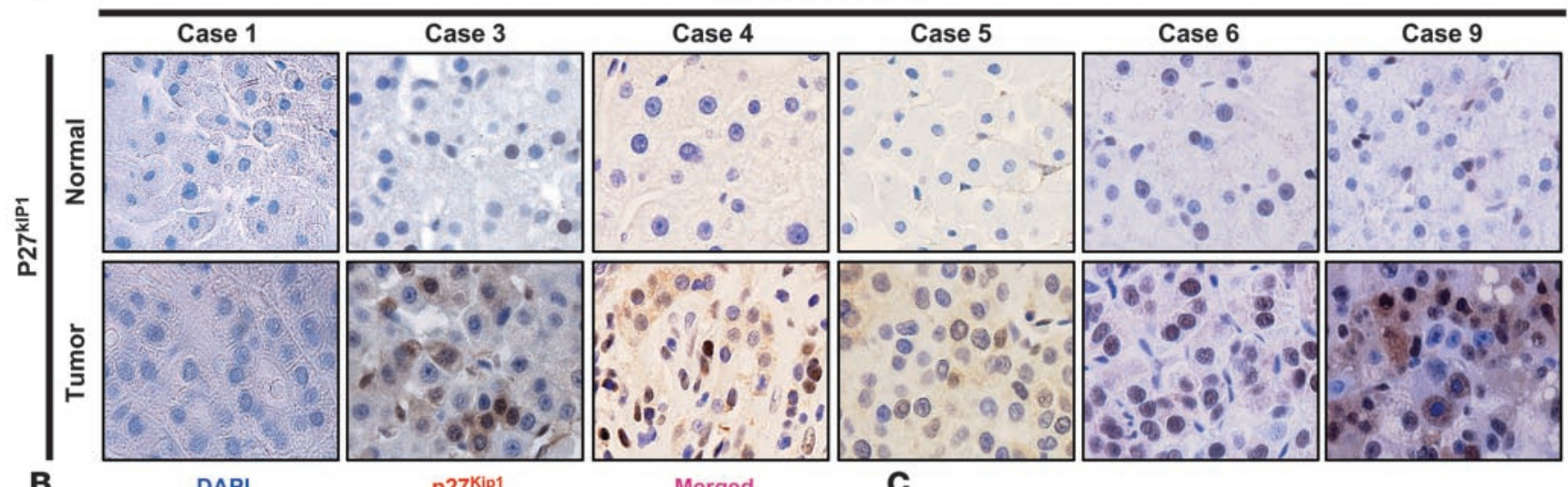

B
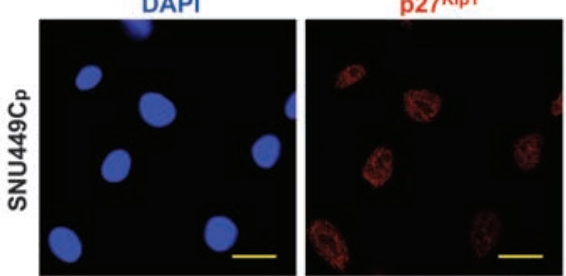

Merged

C

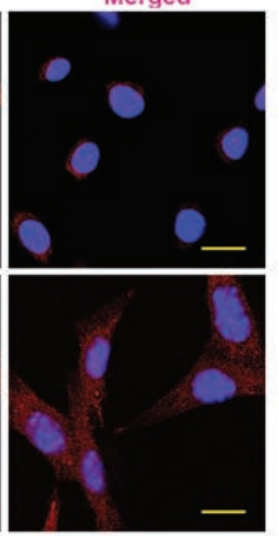

E

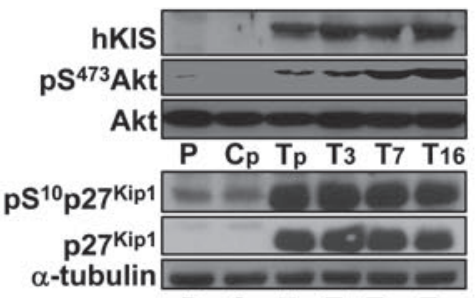

$\begin{array}{lllll}P & \mathrm{~T}_{p} & \mathrm{~T}_{7} & \mathrm{~T}_{16} \mathrm{~T}_{3}\end{array}$ No TM4SF5 TM4SF5 SNU449
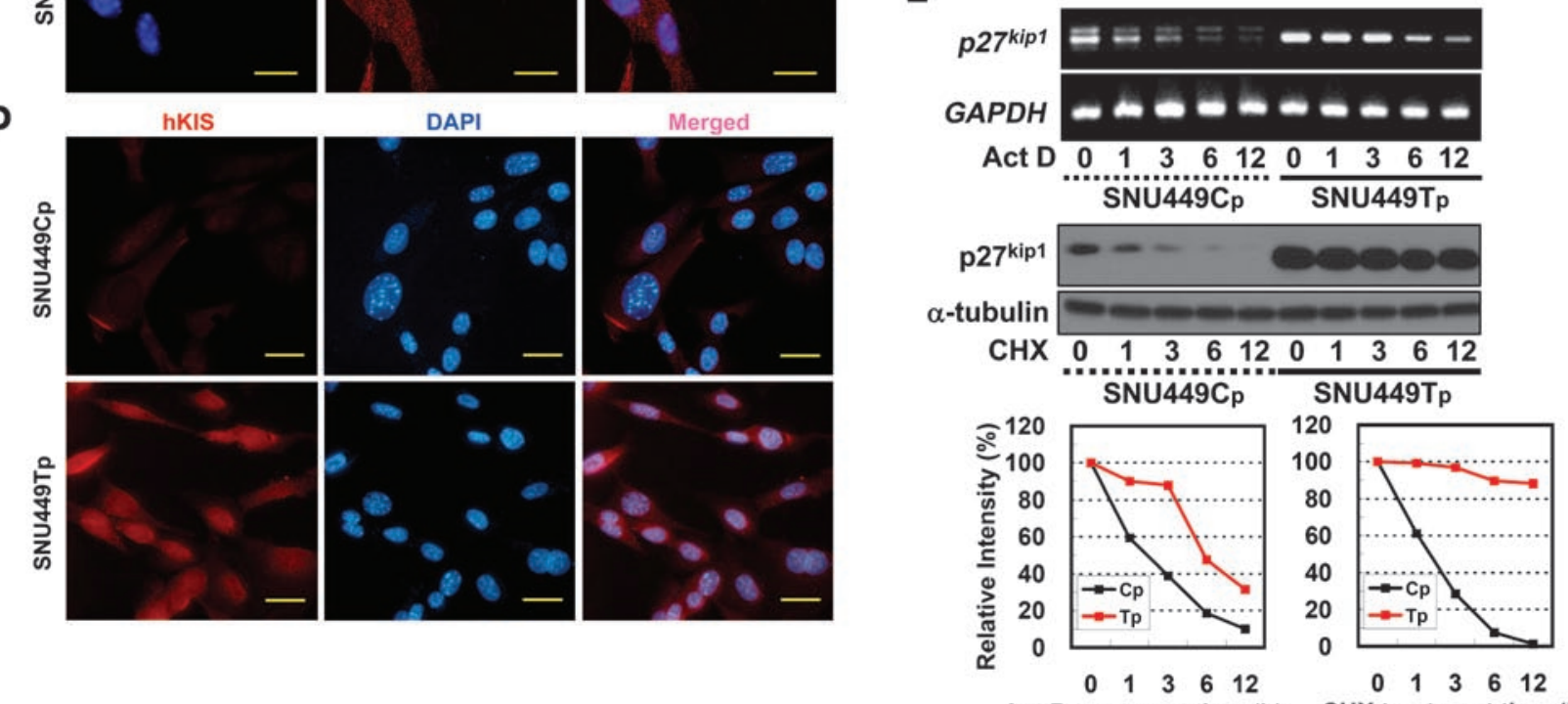

Act $\mathrm{D}$ treatment time (h) $\mathrm{CHX}$ treatment time (h)

Figure 3

TM4SF5 enhanced cytosolic p27 Kip1 stabilization. (A) Representative normal and tumor tissues for immunohistochemistry for p27 Kip1. Original magnification, $\times 400$. SNU449Cp and SNU449Tp were double-stained with DAPI and either anti-p27Kip1 (B) or anti-hKIS (D). (C) Lysates prepared as in Figure 1B were immunoblotted. (E) RT-PCR for $p 27^{K i p 1}$ or GAPDH by using total RNA prepared from actinomycin D-treated cells (Act D, $10 \mu \mathrm{g} / \mathrm{ml}$; upper panel) or immunoblots for $\mathrm{p} 27^{\mathrm{Kip} 1}$ or $\alpha$-tubulin using lysates from cycloheximide treated cells $(\mathrm{CHX}, 10 \mathrm{mM}$; middle panel) were performed. Lower panels: Band intensities were measured, normalized to control band intensities, and plotted. Scale bars: $20 \mu \mathrm{m}$.

polygonal control cell-like morphology without being elongated, whereas untransfected neighboring cells presumably with efficient TM4SF5 expression did not (Figure 2E).

FAKs can regulate RhoA through their guanine-nucleotide exchange factors (GEFs) or GTPase-activating proteins (GAPs) for actin reorganization and morphological changes during cell adhe- sion and spreading (25). Therefore, it is likely that TM4SF5-mediated FAK activation and RhoA inactivation may be interconnected. We thus examined the roles of $\mathrm{PY}^{577} \mathrm{FAK}$ in RhoA inactivation by analyzing FAK association with GAPs via coimmunoprecipitation (Figure 2F). Associations of FAK with p190RhoGAP and/or GTPase regulator associated with FAK (GRAF) were increased in Tp cells as 
A

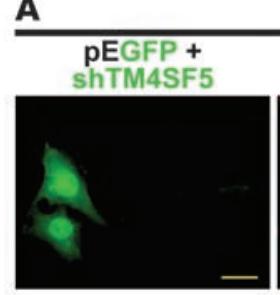

SNU449Tp

C

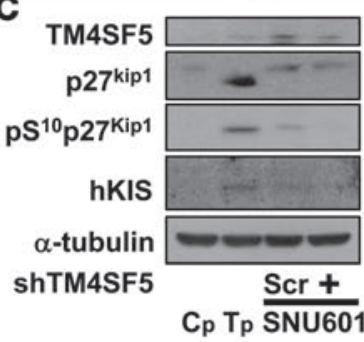
p27 KIP1
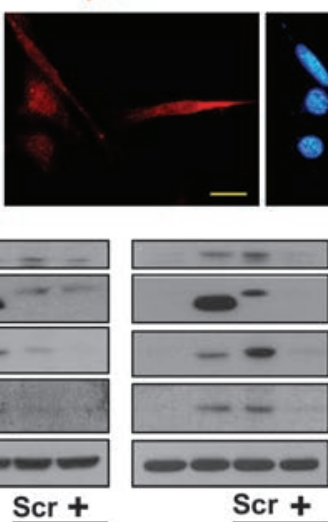

Cp Tp Huh7
DAPI
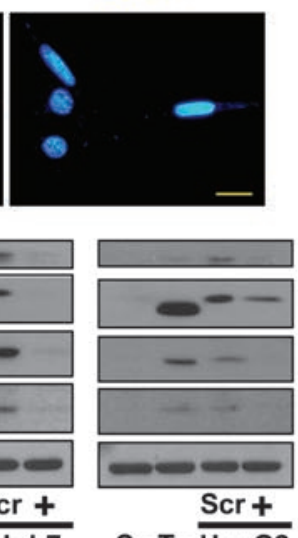

B

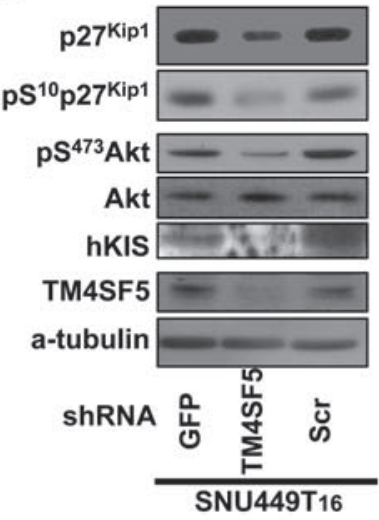

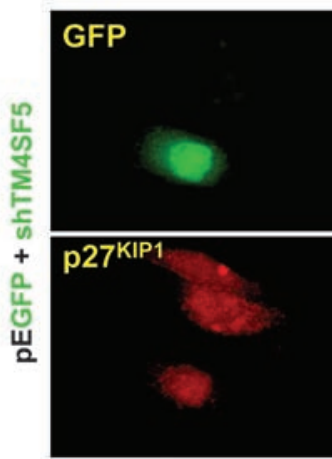

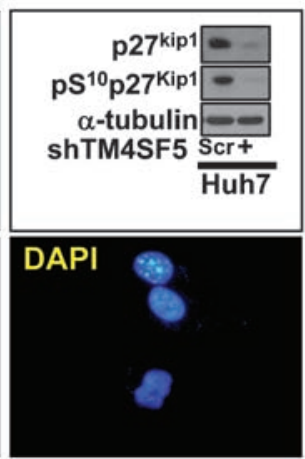

$\mathbf{E}$

Cotransfection of pEGFP and TM4SF5
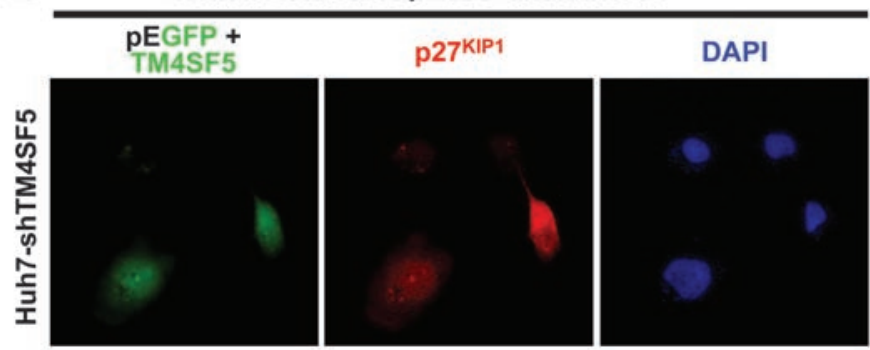

Figure 4

Effects of TM4SF5 suppression on cellular morphology and cytosolic p27kip1 stabilization. (A) SNU449Tp cells were cotransfected with pEGFP and shTM4SF5. After 24 hours, cells were replated on coverslips for an additional 24 hours. Cells were then double-stained for p27kip1 and DAPI. (B) Lysates prepared in Figure 2B were immunoblotted. (C) Huh7, SNU601, and HepG2 cells with endogenous TM4SF5 were transiently transfected with scrambled or shTM4SF5, 48 hours before immunoblots. SNU449Cp and SNU449Tp cells were blotted in parallel for comparison of TM4SF5 expression. (D) Huh7 cells with endogenous TM4SF5 were transiently cotransfected with pEGFP and scrambled sequence or shTM4SF5, 48 hours before immunoblot analysis or immunostaining. Note that shTM4SF5-transfected cells have a lower cytosolic p27Kip1 level, leading to a lower ratio of cytosolic to nucleic p27Kip1 levels. (E) Huh7 cells stably transfected with shTM4SF5 (Huh7-shTM4SF5, Figure 2J) were transiently cotransfected with pEGFP and pcDNA3-TM4SF5. Two days later, the cells were immunostained for p27Kip1 and DAPI. Original magnification, $\times 400$. Data shown represent 3 isolated experiments. Scale bars: $20 \mu \mathrm{m}$.

compared with control Cp cells (Figure 2G). The TM4SF5-enhanced associations between FAK and p190RhoGAP and GRAF were abolished by a mutation of FAK Tyr577Phe (Y577F; Figure 2H), indicating that $\mathrm{pY}^{577} \mathrm{FAK}$ is important for signaling linkages to RhoA via the GAPs in TM4SF5-expressing cells. We next examined whether p190RhoGAP or GRAF might play a role in cellular elongation. Transient expression of GRAF or $\mathrm{p} 190 \mathrm{RhoGAP}$ in control Cp cells caused morphological elongation (Figure 2I). These observations indicate that TM4SF5-mediated morphological elongation and RhoA inactivation were correlated with enhanced FAK/RhoGAPs interactions in SNU449 cells. To avoid a possibility of cloning artifacts, we next examined cells endogenously expressing TM4SF5 for the signaling connection. Using endogenously TM4SF5-expressing Huh7 hepatocarcinoma cells stably transfected with shTM4SF5 (Huh7-shTM4SF5 cells) or scrambled shRNA, we confirmed the positive correlation among $\mathrm{pY}^{577} \mathrm{FAK}, \mathrm{pY}^{416} \mathrm{c}-\mathrm{Src}$, and TM4SF5 (Figure 2J).

TM4SF5-enhanced $27^{\text {Kip } 1}$ expression and cytosolic stabilization. It was previously shown that $\mathrm{p}^{27^{\mathrm{Kip}} 1}$ can translocate to cytoplasm and there bind RhoA, leading to blockade of its GEF binding $(14,26-$ 28). Therefore, we also explored whether TM4SF5-mediated RhoA inactivation correlated with cytosolic $\mathrm{p}^{2} 7^{\mathrm{Kip} 1}$ stabilization. We examined $\mathrm{p} 27^{\mathrm{Kip} 1}$ level and localization first in the hepatocarcinoma tissues. We found, via immunoblotting, that $\mathrm{p} 27^{\mathrm{Kip} 1}$ levels were increased in hepatocarcinoma tissues as compared with normal tissues; 5 cases (cases 1, 3, 4, 5, and 9) of 6 TM4SF5-positive human hepatocarcinoma cases showed enhanced p27 Kip1, and 2 TM4SF5negative cases (cases 2 and 7) did not show increased p27 Kip1 (Figure 1A). In addition, knockdown of endogenous TM4SF5 in Huh7 cells suppressed p27Kip1 expression (Figure 2J). These observations indicate a close correlation between $\mathrm{p} 27^{\mathrm{Kip} 1}$ and TM4SF5 expression levels. Interestingly, 2 $^{\text {Kip1 }}$ immunostains of clinical hepatocarcinoma tissues clearly showed its cytosolic localization in 5 cases (cases 3, 4, 5, 6, and 9; Figure 3A), indicating a quite significant correlation between TM4SF5 and increased expression and cytosolic stabilization of $27^{\text {Kip } 1}$. TM4SF5-expressing cells also showed a prominent $(90 \%)$ cytosolic localization of $\mathrm{p}^{27^{\mathrm{Kip}} 1}$, compared with a 

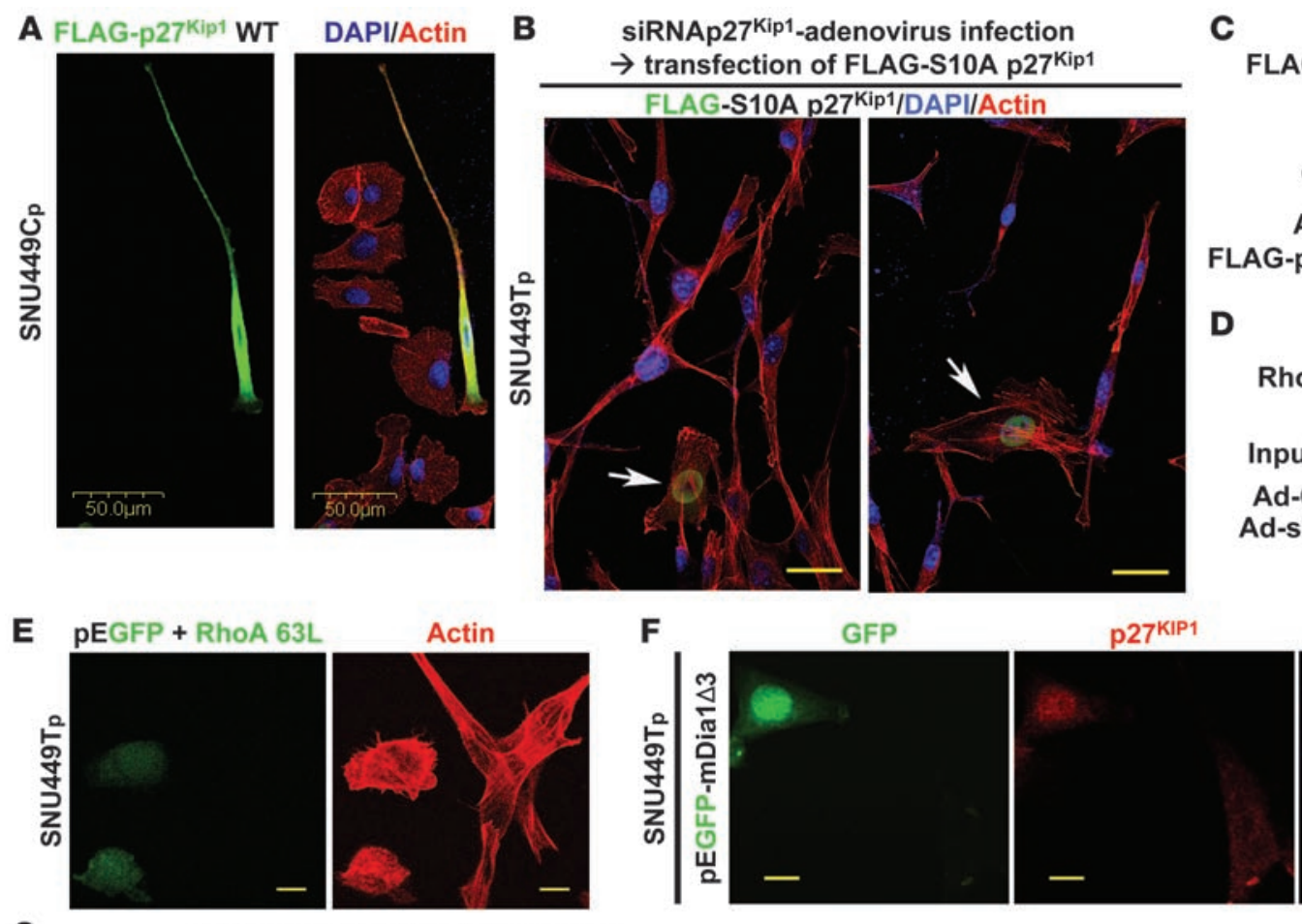

C

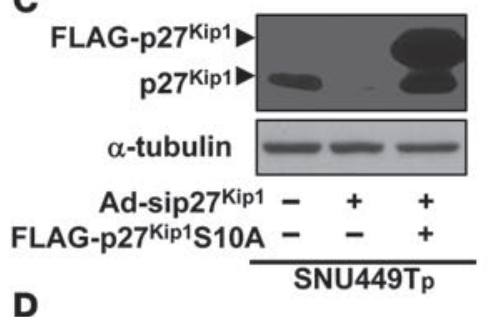

D
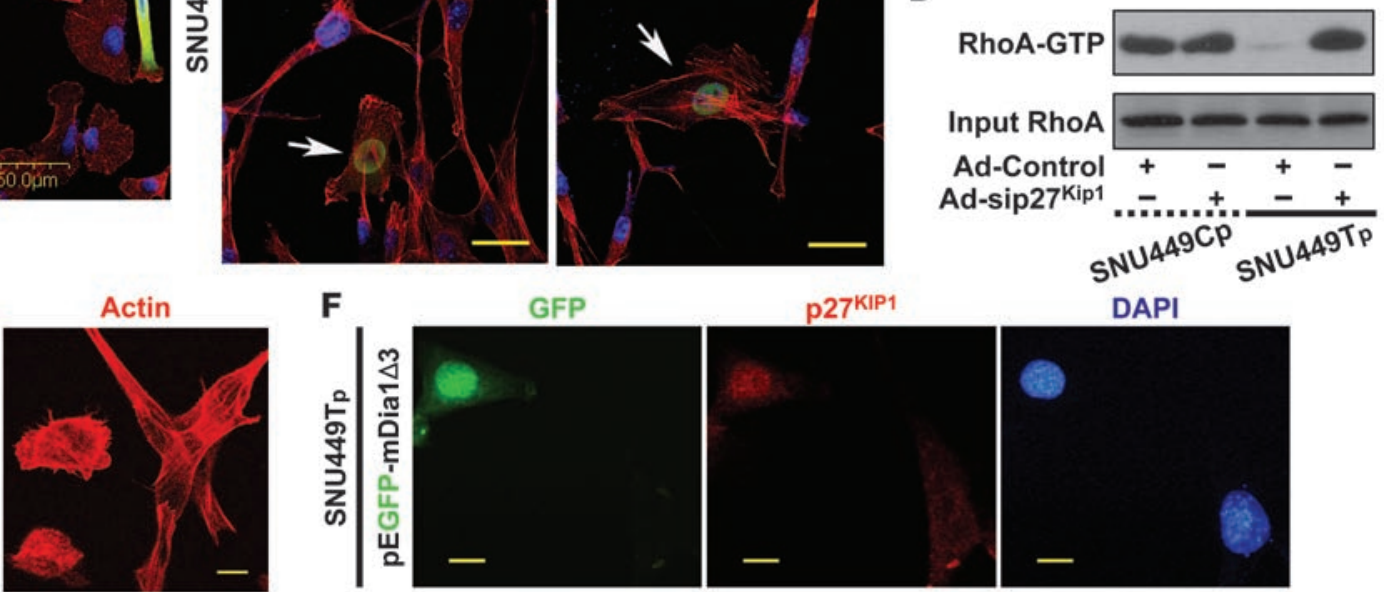

pEGFP $+\mathrm{mDia} 1 \triangle 3$ cotransfection

G

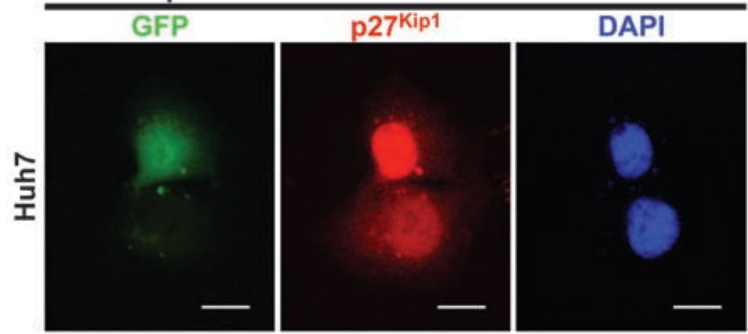

Figure 5

Reciprocal regulation between TM4SF5-mediated cytosolic p27Kip1 stability and Rho GTPase signaling. (A) p27kip1-mediated morphological elongation. SNU449Cp cells were transiently transfected with FLAG-p27kip1 plasmid. After 48 hours, cells were triple-stained with DAPI, phalloidin conjugated with rhodamine, and anti-FLAG- and FITC-conjugated antibody, before confocal microscopic analysis. (B) Reversion of elongated SNMU449Tp cells to control cell-like cells via removal of cytosolic p27Kip1 and concomitant introduction of nuclear p27 Kip1S10A. SNU449Tp cells were infected with siRNAp27 Kip1 adenovirus for 24 hours. Cells were then transfected with FLAG-p27Kip1S10A mutant and incubated for an additional 24 hours, prior to triple-staining, as in A. Note that mutant p27 Kip1-transfected cells (with a green-positive nucleus) have well-developed stress fibers, compared with adjacent untransfected cells. Two images for the same condition are shown. (C) Regulation of p27 ${ }^{\mathrm{Kip} 1}$ levels. Cells, as in B, were immunoblotted. (D) p27Kip1-dependent RhoA activity. Cells were infected with Ad-control or Ad-sip27Kip1 for 24 hours, before being harvested for use in RhoA assay. (E) Reversion of morphological changes by active RhoA. SNU449Tp cells were cotransfected with pEGFP and active RhoA 63L. Actin was stained. (F) RhoA-mediated regulation of cytosolic p27 Kip1 and morphology. SNU449Tp cells were cotransfected with pEGFP plus active mDia (mDia $\triangle 3)$. (G) Huh7 cells were immunostained for DAP1 and p27Kip1, 48 hours after cotransfection with pEGFP and $\mathrm{mDia1} \Delta 3$. Data shown represent 3 independent experiments. Scale bars: $10 \mu \mathrm{m}(\mathbf{E}) ; 20 \mu \mathrm{m}(\mathbf{B}, \mathbf{F}$, and $\mathbf{G}) ; 50 \mu \mathrm{m}(\mathbf{A})$.

primarily nuclear localization in control cells (Figure 3B and Supplemental Figure 2), and showed much enhanced $\mathrm{p} 27^{\text {Kip } 1}$ expression (Figure 3C, lower panel). Since cytosolic p $27^{\mathrm{Kip} 1}$ stabilization was shown to be dependent on Ser10 phosphorylation by KIS (15) or by PKB/Akt (16), their levels were next compared in TM4SF5null or -expressing cells. TM4SF5-expressing cells showed higher Ser10 phosphorylation (i.e., $\mathrm{pS}^{10} \mathrm{p} 27^{\text {Kip } 1}$ ), increased human KIS (hKIS) expression, and increased activity of PKB/Akt ( $\mathrm{pS}^{473} \mathrm{Akt}$ ) (Figure 3C). hKIS expression was prominent in the nucleus and perinuclear areas of TM4SF5-expressing cells and was not apparent in the nucleus or the cytoplasm of the control cells (Figure
3D). When we examined TM4SF5-increased p27Kip1 expression in greater detail, the TM4SF5-enhanced p27 ${ }^{\mathrm{Kip} 1}$ expression level (Figure 3C, lower panel) appeared to result from increased stabilities of its mRNA and protein (Figure $3 \mathrm{E}$ ). We next examined the effects of TM4SF5 knockdown on cellular morphology and cytosolic p27 ${ }^{\text {Kip }} 1$ stabilization. Transient transfection of shTM4SF5 into TM4SF5-expressing cells caused morphological reversion to control cell-like polygonal shapes and reduced cytosolic p27 ${ }^{\mathrm{Kip} 1}$ level (Figure 4A) and furthermore reduced the level of $\mathrm{p} 27^{\mathrm{Kip} 1}$ expression, $\mathrm{pS}^{10} \mathrm{p} 27^{\mathrm{Kip} 1}, \mathrm{pS}^{473} \mathrm{Akt}$, and hKIS expression (Figure 4B). Therefore, these observations indicate that TM4SF5-enhanced $\mathrm{p} 27^{\mathrm{Kip} 1}$ expres- 
A

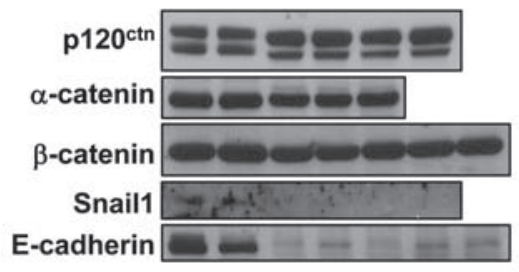

Desmoplakin
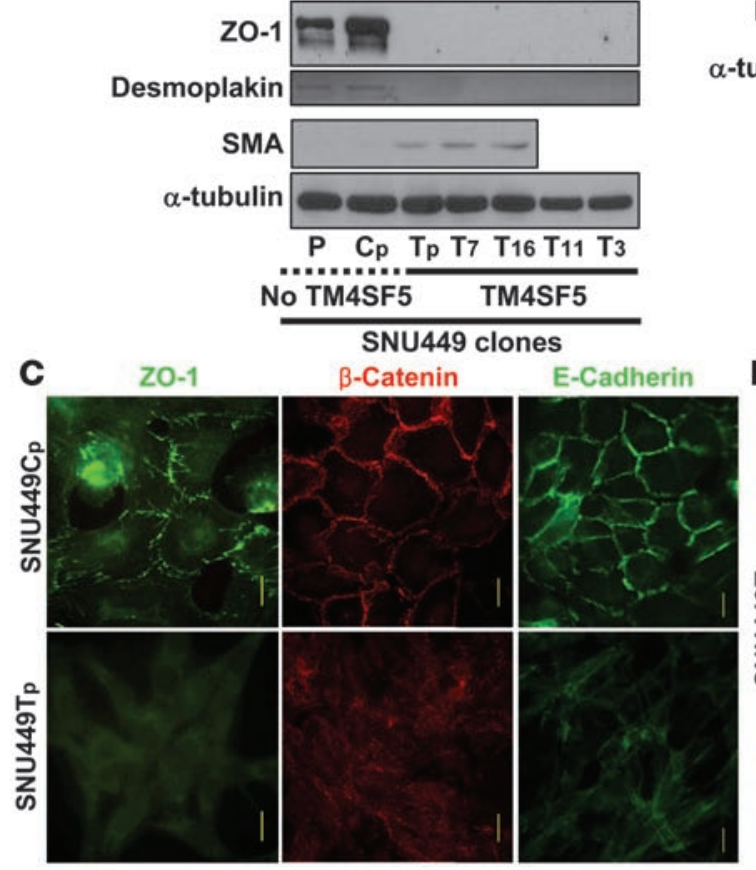

$\mathbf{E}$

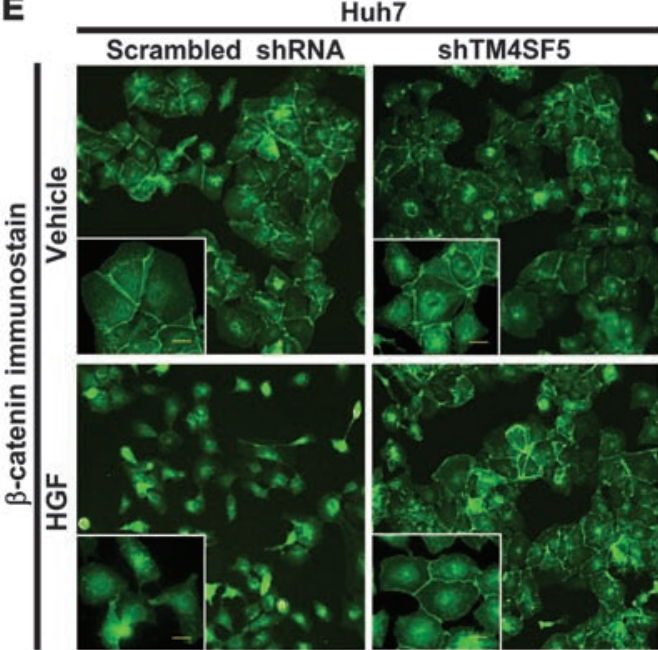

Huh7
B
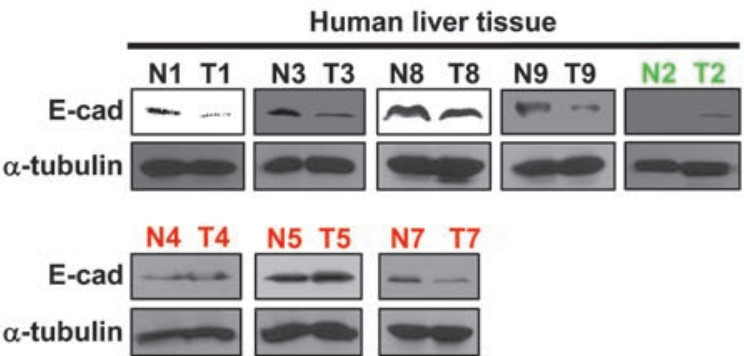
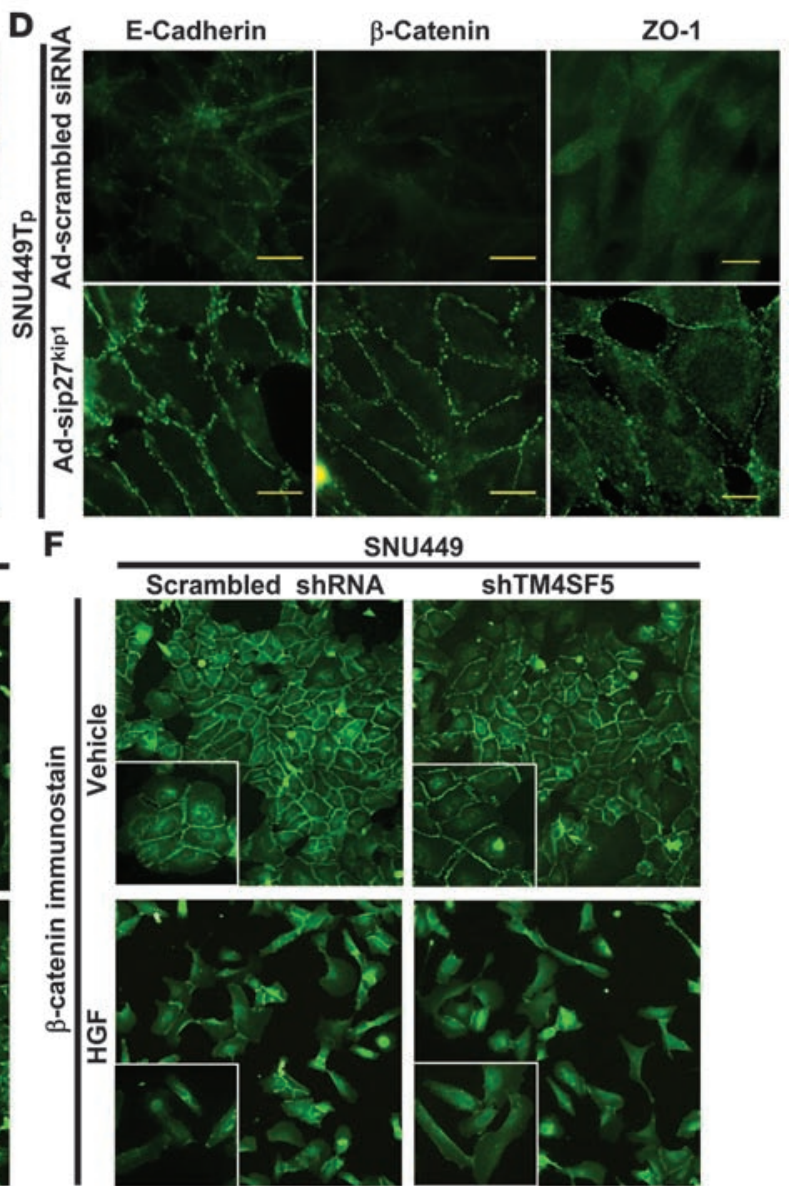

\section{Figure 6}

TM4SF5-enhanced cytosolic p27 Kip1 caused loss of cell-cell contacts. (A) TM4SF5-mediated decrease in certain cell-cell contact molecules. Lysates prepared as in Figure 1C were immunoblotted. (B) Reduced E-cadherin expression in hepatocarcinoma tissues. Lysates from 9 hepatocarcinoma patients were analyzed by standard Western blots using anti-E-cadherin and - $\alpha$-tubulin antibody, as in Figure 1A. Four TM4SF5-positive cases (black letters) and 1 TM4SF5-negative case (green) showed reduced or rather slightly enhanced E-cadherin, indicating a correlation between TM4SF5 expression and E-cadherin suppression. The cases indicated by red letters did not show the correlation. (C) TM4SF5-mediated loss of cell-cell contacts. Confluent SNU449Cp and SNU449Tp cells on coverslips were immunostained for E-cadherin, $\beta$-catenin, or ZO-1. (D) Blockade of TM4SF5-mediated EMT via p27 Kip1 suppression. SNU449Tp cells were infected with adenovirus for scrambled or p27 Kip1 siRNA (Ad-sip27 ${ }^{\text {Kip1 }}$ ), before immunostaining. (E and F) Stable knockdown of endogenous TM4SF5 blocked HGF-mediated EMT of Huh7 cells. Huh7 cells stably transfected with either scrambled shRNA or shTM4SF5 (Huh7-shTM4SF5) (E) or parental SNU449 (F) cells on coverslips were treated with vehicle or HGF $(100 \mathrm{ng} / \mathrm{ml})$ for 24 hours, prior to $\beta$-catenin immunostaining. Original magnification, $\times 400$. Insets are highly magnified images. Data shown represent 3 isolated experiments. Scale bars: $20 \mu \mathrm{m}$. 


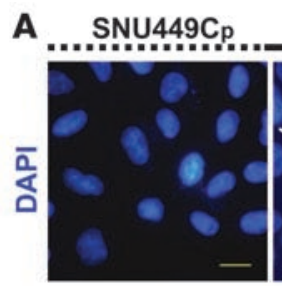

C

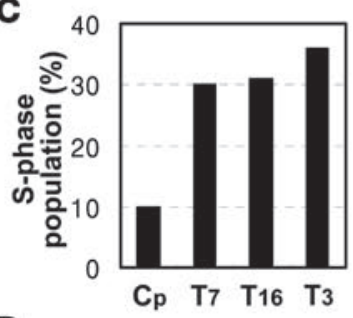

D

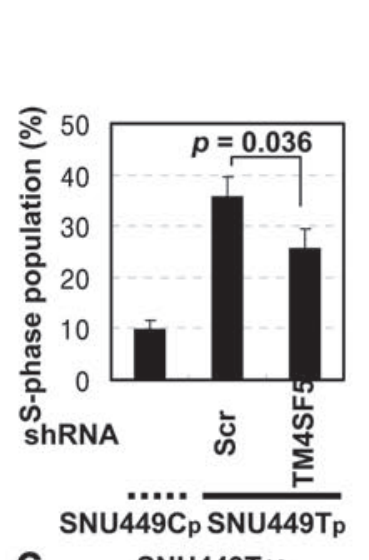

SNU449Tp
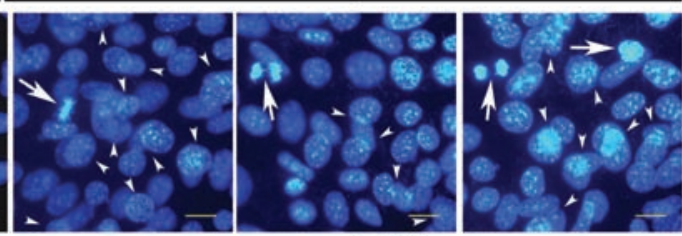

호일 100

है
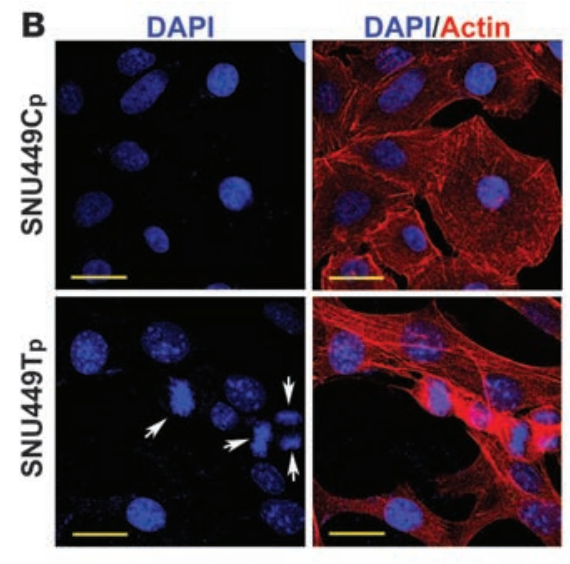

E

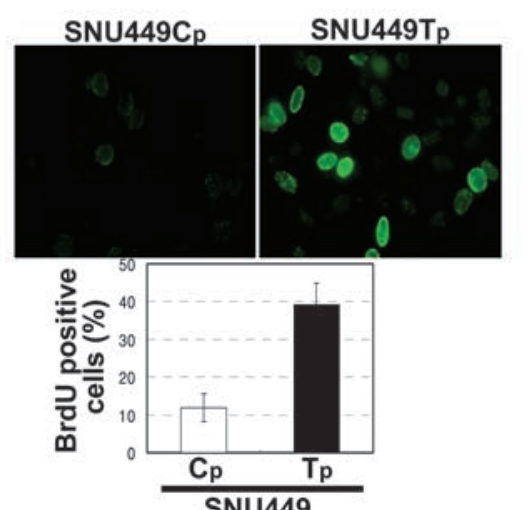

$F$
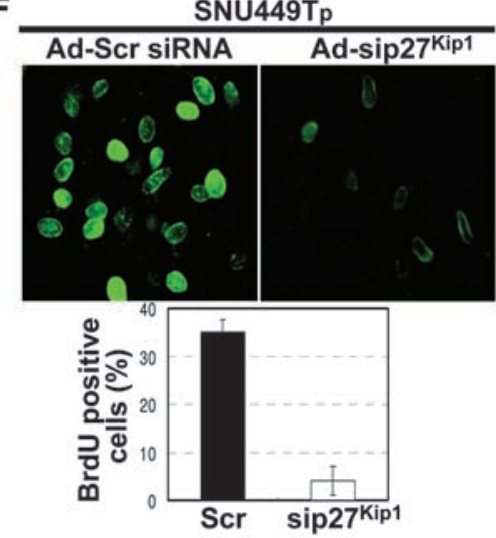

G
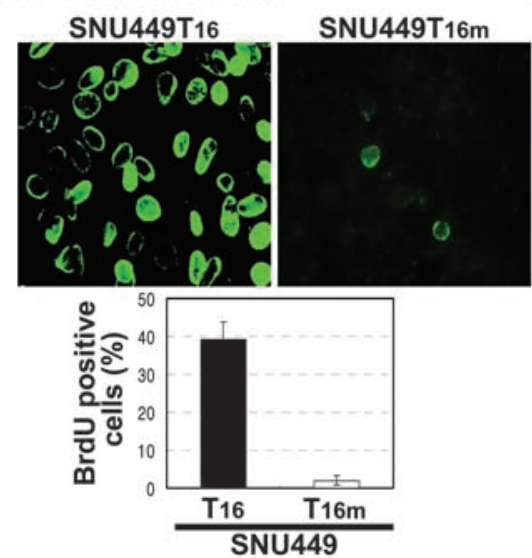

H

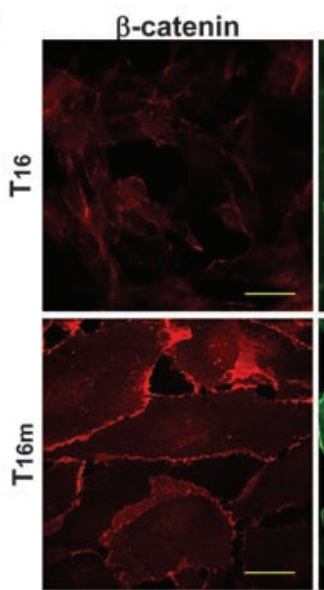

E-cadherin

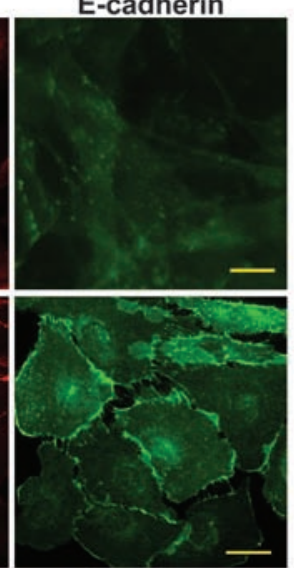

Figure 7

TM4SF5-mediated cytosolic p27Kip1 and EMT caused loss of contact inhibition. (A) Confluent SNU449Cp and SNU449Tp cells on coverslips under normal serum-containing condition were stained with DAPI. Arrows indicate cells in the middle of cell division; arrowheads indicate nuclei overlapped, indicating pile-up of dividing SNU449Tp cells even at confluent conditions. Growth rates of SNU449Cp and SNU449Tp cells were measured by counting viable cells. Each time point represents mean \pm SD of an experiment performed in triplicate. (B) Subconfluent SNU449Cp and SNU449Tp cells as in A were double-stained for DNA and actin and visualized by a confocal microscope. Arrows indicate superimposed nuclei in the middle of cell division even in a subconfluent condition. S-phase populations of SNU449Cp and diverse TM4SF5-expressing cell lines (T7, T16, and T3) (C) or SNU449Cp and SNU449Tp cells transfected with scrambled or shTM4SF5 (D) were stained with propidium iodide before DNA content analyses using flow cytometry. SNU449Cp and SNU449Tp cells (E), SNU449Tp cells infected with scrambled (Ad-Scr) or p27Kip1 (Ad-sip27Kip1) siRNA adenovirus (F), or SNU449T16 cells reconstituted without (SNU449T16) or with E-cadherin (SNU449T16m) (G) were analyzed for S-phase progression via BrdU incorporation assay. Values in graphs represent mean \pm SD. Original magnification, $\times 400$. (H) Confluent SNU449T16 (T16) and SNU449T16m (T16m) cells on coverslips were immunostained. Data represent 3 independent experiments. Scale bars: $20 \mu \mathrm{m}$. 
sion and cytosolic stabilization are linked to morphological elongation. Interestingly, the FAK Y577F mutation did not affect total $\mathrm{p} 27^{\mathrm{Kip} 1}$ and $\mathrm{pS}^{10} \mathrm{p}^{2} 7^{\mathrm{Kip} 1}$ levels, indicating that TM4SF5-mediated RhoA inactivation might be correlated separately with 2 distinct pathways, the first via FAK/RhoGAP associations and the second involving cytosolic p27 ${ }^{\mathrm{Kip} 1}$ (Supplemental Figure 3 and Figure 2F). TM4SF5-mediated cytosolic p27 ${ }^{\text {Kip } 1}$ stability was examined in hepatoma cells endogenously expressing TM4SF5. Knockdown of endogenous TM4SF5 via shTM4SF5 transfection reduced p27Kip1 expression, $\mathrm{pS}^{10} \mathrm{p} 27^{\mathrm{Kip} 1}$, and hKIS levels (Figure $4 \mathrm{C}$ ) and presumably facilitated degradation of cytosolic, but not nuclear, $\mathrm{p} 27^{\mathrm{Kip} 1}$ (Figure 4D). Furthermore, reexpression of TM4SF5 in Huh7-shTM4SF5 cells appeared to increase cytosolic p27Kip1 (Figure 4E). These observations suggest that TM4SF5 enhanced $\mathrm{p} 27^{\mathrm{Kip} 1}$ expression and cytosolic stabilization.

Cytosolic p $27^{\text {Kip } 1}$ mediates inactivation of RhoA and elongation of TM4SF5-expressing cells. Since TM4SF5-expressing cells were elongated and expressed more $\mathrm{p} 27^{\mathrm{Kip} 1}$, we tested whether transfection of p27 Kip1 in TM4SF5-null cells caused morphological elongation. Introduction of $\mathrm{p} 27^{\mathrm{Kip} 1}$ caused transfected cells to be elongated as compared with neighboring untransfected control cells (Figure $5 \mathrm{~A})$. By contrast, knockdown of $\mathrm{p} 27^{\mathrm{Kip} 1}$ together with transfection

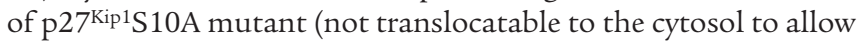
$\mathrm{p} 27^{\text {Kip } 1}$ to accumulate in the nucleus) in TM4SF5-expresing cells resulted in a control cell-like polygonal morphology with obvious stress fibers (Figure 5, B and C). We next explored a possible role of (cytosolic) $\mathrm{p}^{27^{\mathrm{Kip}} 1}$ in TM4SF5-mediated RhoA inactivation. TM4SF5-expresing cells with an insignificant RhoA activity showed a recovery of RhoA activity by knockdown of p27kip1, whereas TM4SF5-null control cells sustained it at a significant level (Figure 5D). This observation indicates that $\mathrm{p} 27^{\mathrm{Kip} 1}$ expression may lead to inhibition of RhoA activity, as expected (Figure 2F; refs. 14, 27-29). Interestingly, active RhoA or active mDia ( $\mathrm{mDia} \Delta 3$, a RhoA downstream effector) transfection or treatment with lysophosphatidic acid (LPA, an activator of RhoA signaling) converted the elongated morphology to a control cell-like one and reduced cytosolic p $27^{\mathrm{Kip} 1}$ (Figure 5, E and F, and Supplemental Figure 4). It might thus be likely that cytosolic $\mathrm{p} 27^{\mathrm{Kip} 1}$ was no longer stabilized when RhoA pathway signaling was activated (Figure $5 \mathrm{~F})$. Introduction of active $\mathrm{mDia} 1 \Delta 3$ into Huh7 cells resulted in less cytosolic (or more nuclear) p2 $7^{\mathrm{Kip} 1}$ (Figure $5 \mathrm{G}$ ). These observations indicate that there appeared to be a linkage between cytosolic $\mathrm{p}^{27^{\mathrm{Kip}} 1}$ level and RhoA activity, which might be involved in TM4SF5-mediated morphological elongation.

TM4SF5-enhanced cytosolic p27Kip1 causes EMT. We next wondered about the biological significance of TM4SF5-mediated cell elongation. It is possible that TM4SF5 expression-mediated morphological elongation with aberrant actin organization (Supplemental Figure 1) may affect cell-cell adhesion status, since actin reorganization at the cell periphery could regulate cell-cell contacts (30). To test the possibility, we first immunoblotted the molecules that are involved in cell-cell contact formation. Although TM4SF5-null cells showed easily detectable levels of the molecules we tested, TM4SF5-expressing cells showed decreased E-cadherin, ZO-1, and desmoplakin but increased $\alpha$-SMA levels, indicating a TM4SF5-mediated EMT (Figure 6A). Interestingly, the decrease in E-cadherin was not accompanied by an increase in Snail1, a known transcriptional suppressor of E-cadherin, but instead Snail1 was also decreased in TM4SF5-expressing cells (Figure 6A). This observation indicates that E-cadherin is regulated in a Snail1- independent manner and instead in a TM4SF5-mediated manner in this system. Importantly, the TM4SF5 overexpression tendency was also correlated with loss of E-cadherin expression in clinical hepatocarcinoma samples; the TM4SF5-overexpressing cases (i.e., cases 1, 3, 8, and 9) and -null case (case 2) showed reduced or rather enhanced E-cadherin expression, respectively (Figure $6 \mathrm{~B})$. This observation indicates that the tumor progression of certain liver cancer patients might involve TM4SF5-mediated loss of E-cadherin expression, although not all cases showed the correlation (e.g., cases 4, 5, and 7), and how TM4SF5 caused loss of E-cadherin expression is currently unknown. We next immunostained molecules at cell-cell contacts to see whether TM4SF5 might cause loss of cell-cell contacts. Immunostaining showed reduced localization of E-cadherin, $\beta$-catenin, and ZO- 1 at cell-cell contacts of TM4SF5-expressing cells as compared with control cells (Figure 6C). Since TM4SF5-mediated morphological elongation depended on increased cytosolic p27 ${ }^{\mathrm{Kip} 1}$, we next examined whether knockdown of $\mathrm{p}^{27^{\mathrm{Kip}} 1}$ using an siRNA adenovirus might cause reformation of cell-cell contacts in TM4SF5-expressing cells. Interestingly, p27 ${ }^{\text {Kip1 }}{ }^{1}$ knockdown resulted in maintenance of cell-cell contacts between TM4SF5-expressing cells (Figure 6D) and reduction of $\alpha$-SMA (data not shown). To reveal the roles of endogenous TM4SF5 in induction of EMT, hepatocyte growth factor-mediated (HGF-mediated) EMT was analyzed using Huh7 cells with (i.e., parental Huh7) or without TM4SF5 (i.e., Huh7-shTM4SF5; Figure 2J). When treated with HGF, Huh7 cells transfected with control shRNA showed EMT, whereas Huh7-shTM4SF5 cells did not (Figure 6E), indicating that TM4SF5 mediates EMT. Meanwhile, stable transfection of shTM4SF5 into TM4SF5-null SNU449 cells did not affect HGF-mediated EMT (Figure 6F).

TM4SF5-mediated loss of contact inhibition. During EMT analysis via immunostaining of confluent cells, we often found that confluent TM4SF5-expressing cells showed overlapped nuclei, including ones in the middle of the cell division process, whereas confluent control cells did not (Figure 7A). It is likely that TM4SF5 mediates contact inhibition loss. We next studied whether positioning of TMS4SF5-expressing cells was multilayered even at a subconfluent condition. When actin was stained, TM4SF5-expressing cells showed overlapped nuclei in the middle of cell division even in a subconfluent condition, whereas control cells showed wellestablished cell-cell contacts and did not pile up (Figure 7B). Meanwhile, growth of $\mathrm{T} p$ cells was uncontrolled and continued at increasing cell density, but growth of $\mathrm{Cp}$ cells reached saturation presumably by contact inhibition (Figure 7A, graph). These observations may suggest that confluent TM4SF5-expressing cells lost contact inhibition by virtue of inability to sense neighboring cells. When we next analyzed the DNA contents of the cells at a confluent condition, we found that static S-phase populations in TM4SF5-expressing cells were much higher than those in control cells (Figure 7C). When TM4SF5 was reduced by shTMSF5 transfection, the static S-phase population was reduced significantly, by about $30 \%$, consistent with transfection efficiency of around $30 \%-40 \%$ (Figure 7D). Furthermore, TM4SF5-expressing cells showed a higher S-phase progression rate than control cells (Figure 7E). We next examined whether blocking of the TM4SF5 effects might abolish the enhanced S-phase progression of TM4SF5-expressing cells. TM4SF5-enhanced S-phase progres-

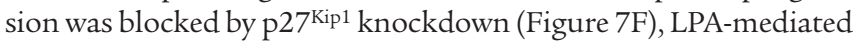
RhoA activation (Supplemental Figure 5), or E-cadherin reexpression (Figure 7G), indicating that TM4SF5-expressing cells could 
A

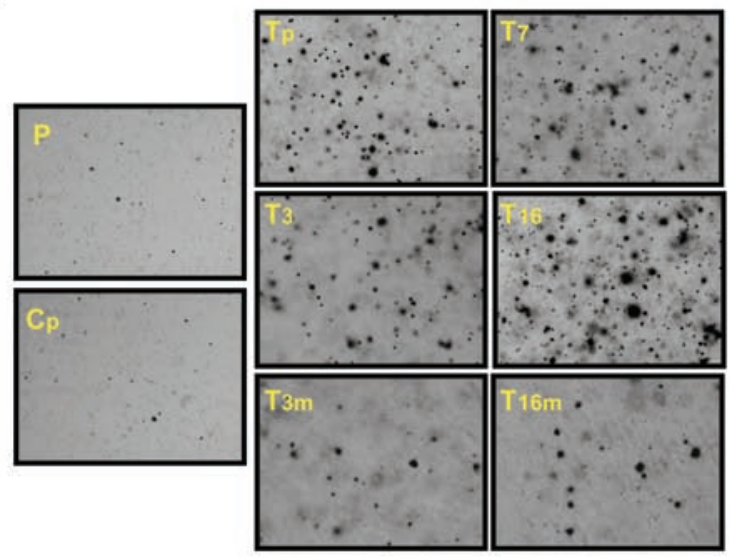

B
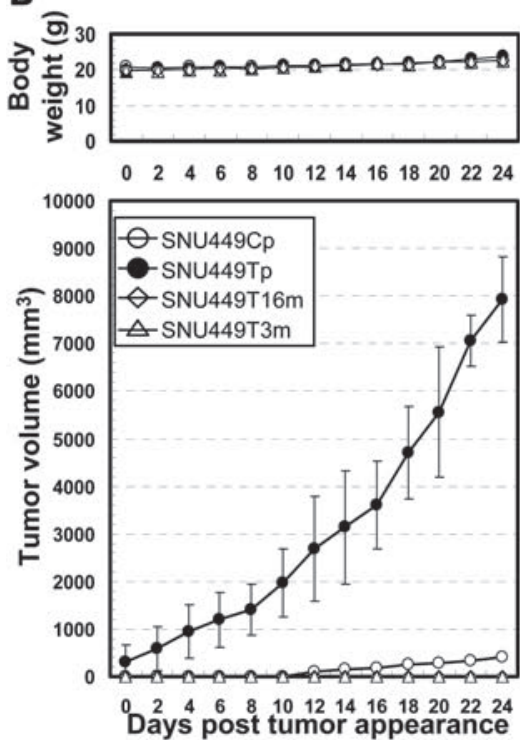

D

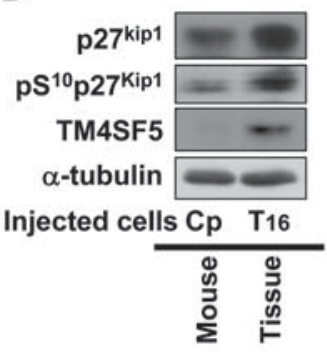

C

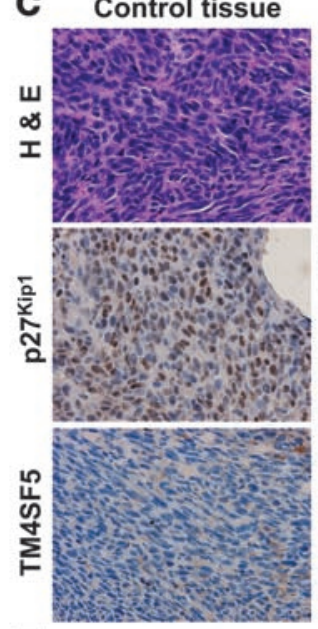

E

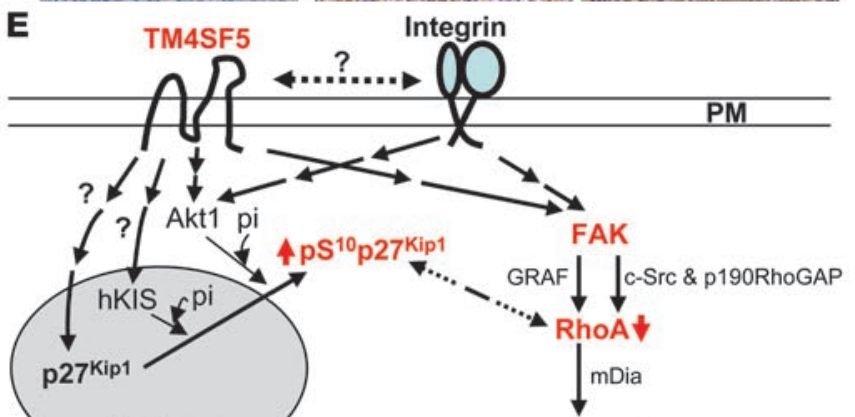
\& morphological elongation

Cytosol
Actin organization

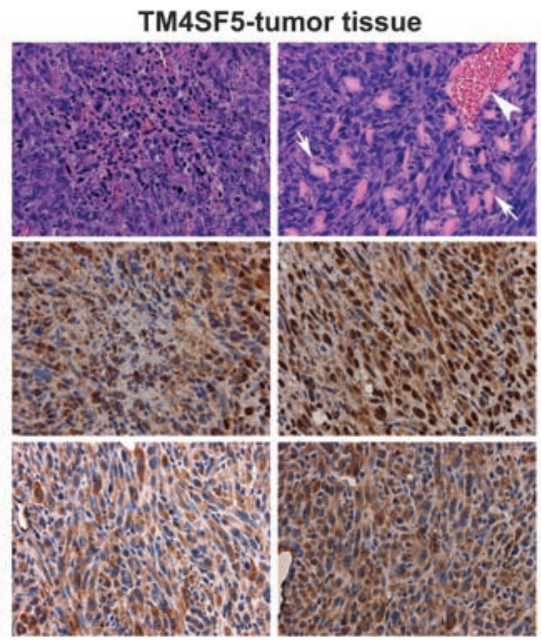

E-cadherin $\downarrow$ T SMA

Loss of Contact Inhibition

Figure 8

TM4SF5-mediated tumorigenesis. (A) Soft agar assay of SNU449 cells was performed for 27 days. (B) Cells were injected subcutaneously to mice $\left(1 \times 10^{7}\right.$ cells/mouse). Tumor volume values and body weights were measured as described in Methods and plotted as mean \pm SD. Values for SNU449T16m and SNU449T3m were zero, since they did not form tumors in mice. (C) Tumors from SNU449Cp or T16 cell-injected mice were stained for H\&E or for p27 Kip1 or TM4SF5. Original magnification, $\times 40$ (A); $\times 100$ (C). (D) Extracts from control or TM4SF5-expressing cell-injected mice were immunoblotted for p27 Kip1, pS ${ }^{10}$ p2 $7^{\text {Kip1 }}$, and TM4SF5. (E) Working model of TM4SF5-mediated EMT and contact inhibition loss. Overexpression of TM4SF5 appears to enhance p27 Kip1 expression and cytosolic stabilization, being correlated with p27 Kip1 Ser10 phosphorylation, presumably by hKIS and/or PKB/Akt. TM4SF5 also affects FAK signaling to RhoA via RhoGAPs. These TM4SF5 effects indicate a linkage between cytosolic p27Kip1 expression and stabilization and RhoA activity regulation, which may modulate cellular morphology. Morphological change in turn causes EMT, which involves E-cadherin suppression and $\alpha$-SMA induction in TM4SF5-expressing cells. Due to cell-cell contact loss, one cell may not recognize adjacent cells and grow in an uncontrollable manner, leading to contact inhibition loss (i.e., piling up of cells). Solid-line connections with question mark indicate that it is currently unknown how TM4SF5 exerts its effects, and dotted lines indicate either a cross-talk between the 2 receptors not evidenced but prospective from another report (23) or a functional relationship between 2 parties with unknown mechanisms. pi, inorganic phosphate.

more effectively progress through the S-phase of the cell cycle via TM4SF5-mediated effects. Upon reexpression of E-cadherin in TM4SF5-expressing T16 cells, there was reduced $\alpha$-SMA expression (Supplemental Figure 6) and recovered cell-cell contacts (Figure 7H). Thus, the observations with TM4SF5 cells reconstituted with E-cadherin (i.e., SNU449T16m) indicate that TM4SF5-mediated EMT led to contact inhibition loss, suggesting a close functional linkage between cell-cell contact and contact inhibition.
TM4SF5-mediated tumorigenesis. We next examined tumorigenicity of TM4SF5 via 2 approaches. First, SNU449 cell lines with or without TM4SF5 were investigated for anchorage-independent growth. After 27-day culture in soft agar, TM4SF5-expressing cells clearly formed more foci, compared with control cells (Figure 8A). However, TM4SF5-expressing cells reconstituted with E-cadherin (SNU449T3m and SNU449T16m cells) showed much-reduced foci formation, compared with their TM4SF5-expressing counterparts 
(SNU449T3 and SNU449T16 cells, respectively; Figure 8A). Therefore, TM4SF5-mediated EMT appeared to correlate with anchorage-independent growth. To examine TM4SF5-mediated tumor formation in nude mice, SNU449 cells were subcutaneously injected into mice, and then tumor sizes were evaluated to calculate tumor volumes every other day for 6 weeks. Whereas parental, Cp cells did not form substantial tumors, TM4SF5-expressing SNU449T16 cells did ( $n=6$ for each case); injection of TM4SF5-expressing cells formed tumors $\left(n=6 ; 7,051.5 \pm 534.6 \mathrm{~mm}^{3}\right)$ growing continuously, whereas control TM4SF5-null cell injection resulted in 1 mouse with a small tumor $\left(<200 \mathrm{~mm}^{3}\right)$ and 5 other mice without any tumor (Figure 8B). Meanwhile, SNU449T16m or SNU449T3m cell-injected mice ( $n=6$ for each case) did not form tumors (Figure $8 \mathrm{~B})$. Furthermore, all mice showed normal increases in body weight (Figure 8B, upper panel). Immunohistochemistry of the tumor tissues showed substantial necrotic cell populations in the middle of the TM4SF5-mediated tumors (Figure 8C, upper middle panel) and tumor cells invaded through local cytoskeletal muscles (arrows) and blood vessels (arrowhead) (Figure 8C, upper right panel). In addition, cytosolic p $27^{\text {Kip1 }}$ localization or TM4SF5 in TM4SF5-mediated tumors was prominent, compared with that in epidermal tissue section from control cell-injected mice (Figure 8C, lower panels). Furthermore, immunoblots showed that TM4SF5, p27 Kip1, and $\mathrm{pS}^{10} \mathrm{p} 27^{\text {Kip } 1}$ levels were much higher in TM4SF5-mediated tumors than in control tissue (Figure 8D).

\section{Discussion}

This study provides evidence that TM4SF5 is an inducer of EMT that eventually leads to uncontrolled human hepatocarcinoma cell growth in both cell culture and in vivo systems. Expression of TM4SF5 in hepatocyte cells caused increased $\mathrm{p} 27^{\mathrm{Kip} 1}$ expression and cytosolic stabilization, morphological elongation, cell-cell contact loss, continuous growth, uncontrolled S-phase progression, and anchorage-independent growth, and injection of TM4SF5expressing cells into nude mice formed tumors (Figure 8E).

The tumorigenic functions of TM4SF5 at the molecular level have been unknown so far, although it was shown to be highly expressed in diverse tumor types $(21,22)$, including liver carcinoma (this study). Histological differences were not observed between the TM4SF5-positive and -negative tissues (data not shown), and mutations in the TM4SF5 gene leading to tumorigenesis are not known so far. Other TM4SFs are known to cooperate with integrins in their roles in cell proliferation, adhesion, and migration (4, 5 ). We recently reported that TM4SF5-mediated actin reorganization and focal adhesion turnover in Cos7 cells were regulated positively by $\alpha_{2}$ integrin but negatively by serum treatment and that TM4SF5 association with $\alpha_{2}$ integrin was abolished by growth factor stimulation, suggesting that TM4SF5 can regulate architectural organization and function of membrane receptors such as integrins (23). Therefore, it is likely that TM4SF5 in epithelial cells affected activities of FAK and RhoA, which are major downstream effectors of integrins, presumably via cooperation with integrins and/or integrin-mediated signaling.

In this study, a close correlation between TM4SF5 overexpression and enhanced $\mathrm{p}^{2} 7^{\mathrm{Kip}} \mathrm{1}$ expression and cytosolic stabilization was obvious in analysis of liver tissues from hepatocarcinoma patients. $27^{\text {Kip } 1}$ acts as an inhibitor of CDKs in the nucleus during cell-cycle progression (31). Since TM4SF5-expressing cells showed an enhanced stability of $\mathrm{p} 27^{\mathrm{Kip} 1} \mathrm{mRNA}$ and protein, a shortened doubling time (Figure 7A), and an enhanced S-phase cell-cycle pro- gression leading to uncontrolled cell growth, it may be likely that p27 ${ }^{\text {Kip }}{ }^{1}$ overexpressed in TM4SF5-expressing cells might have roles in cytosol besides just cell-cycle inhibition in nucleus. Cytosolic stabilization of $\mathrm{p}^{27^{\mathrm{Kip}} 1}$ was previously shown in diverse tumors (17-19), although its significance in uncontrolled cell growth is not completely understood. $27^{\mathrm{Kip} 1}$ translocates to the cytosol subsequent to Ser10 phosphorylation by KIS (15) or PKB/Akt (32). hKIS was much more highly expressed and enriched in the nucleus and perinuclear areas of TM4SF5-expressing cells, compared with its insignificant level in the cytosol of TM4SF5-null cells, although it is currently unknown how TM4SF5 enhanced it. TM4SF5-expressing cells also showed enhanced Akt (Ser473) phosphorylation, compared with TM4SF5-null control cells. Therefore, hKIS induced and Akt activated by TM4SF5 were correlated with the cytosolic $\mathrm{p} 27^{\mathrm{Kip} 1}$ localization. In addition, $\mathrm{p} 27^{\mathrm{Kip} 1}$ is known to be additionally phosphorylated at Thr187 and Thr157, resulting in cytosolic stabilization $(33,34)$. In this study, however, $\mathrm{pT}^{157} \mathrm{p} 27^{\mathrm{Kip} 1}$, and $\mathrm{pT}^{187} \mathrm{p} 27^{\mathrm{Kip} 1}$ were not correlated with TM4SF5 expression (data not shown). Thus, TM4SF5-enhanced cytosolic p27 ${ }^{\text {Kip } 1}$ might involve its Ser10 phosphorylation in our system, being consistent with the recent report that transgenic mice with the $\mathrm{p} 27^{\mathrm{Kip} 1} \mathrm{~S} 10 \mathrm{~A}$ mutation were refractory to Ras-induced cytoplasmic translocation of $\mathrm{p}^{2} 7^{\mathrm{Kip} 1}$ (28). Thus, it is likely that TM4SF5 may be involved in cytosolic p27 ${ }^{\mathrm{Kip} 1}$ stabilization observed in diverse tumors (17-19), including hepatocarcinoma (this study). Although it is unknown how TM4SF5 induced $\mathrm{p} 27^{\mathrm{Kip} 1}$, the current study showed that TM4SF5 expression correlated with more p27 ${ }^{\text {Kip }} 1$ in cytosol, RhoA inactivation, and morphological elongation. We also found that $\mathrm{p} 190 \mathrm{RhoGAP}$ and GRAF appeared to be involved in the TM4SF5-mediated morphological elongation via

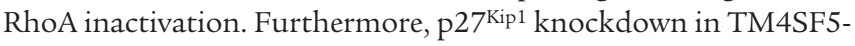
expressing cells resulted in recovery of RhoA activity and of control cell-like morphology. Therefore, there appears to be a linkage between cytosolic $\mathrm{p}^{27^{\mathrm{Kip}} 1}$ stabilization and RhoA activity.

Interestingly, TM4SF5 expression resulted in enhanced $\alpha$-SMA expression and EMT, which were abolished by $\mathrm{p}^{27^{\mathrm{Kip}} 1 \mathrm{knockdown}}$ in TM4SF5-expressing cells. The TM4SF5-mediated EMT involved E-cadherin suppression in a Snail1-independent manner, but instead in a TM4SF5-mediated manner. In addition, TM4SF5 expression was significantly correlated with reduced E-cadherin expression in clinical hepatocarcinoma liver tissues. These observations suggest that the TM4SF5-mediated tumorigenesis may involve an EMT via reduced E-cadherin expression. It was previously reported that RhoA activation was required for $\alpha$-SMA expression and EMT, when mammary epithelial cells were treated with TGF- $\beta 1$ (35). However, RhoA activity in mouse myoblasts was also previously shown to be required for $\beta$-catenin localization at cell-cell contact sites (36). Although it is thus likely that the role of RhoA activity in cell-cell contacts depends on cell type and signaling context, we can speculate that TM4SF5 expression caused not only RhoA inactivation sufficient for dramatic reorganization of actin filaments linked to cell-cell contacts via cadherin/catenin plaque complexes but also E-cadherin expression loss, leading to EMT. It was interesting to observe that $\mathrm{p} 27^{\mathrm{Kip} 1}$ knockdown blocked EMT of TM4SF5-expressing cells, although p27 Kip1 knockdown and concomitant nuclear p27 ${ }^{\text {Kip } 1}$ accumulation were required for morphological reversion from elongated cells to polygonal control cell-like cells. The difference may indicate differential significance of the ratio of $\mathrm{p} 27^{\mathrm{Kip} 1}$ in the nucleus to that in the cytosol for morphology and for cell-cell contact formation. Furthermore, knockdown of endogenous TM4SF5 in Huh7 cells reduced 
$\mathrm{p} 27^{\mathrm{Kip} 1}$ and $\mathrm{pS}{ }^{10} \mathrm{p} 27^{\mathrm{Kip} 1}$ and blocked HGF-mediated loss of cell-cell contacts, indicating that TM4SF5 causes EMT in certain signaling contexts involving cytosolic 2 27Kip1.

This study also revealed for the first time to our knowledge that TM4SF5 caused loss of contact inhibition, presumably through an EMT, leading to uncontrolled growth in multilayers and in soft agar and to tumorigenesis in nude mice. E-cadherin-mediated cell-cell contacts were shown to suppress proliferation and tumorigenesis $(37,38)$. It was recently reported that decreased cell spreading during contact inhibition may be responsible for growth arrest (39). Alternatively, loss of cell-cell contacts may cause one cell not to recognize adjacent cells and thereby divide as frequently as possible, leading to loss of contact inhibition. Consistent with this idea, superimposed nuclei were observed in confluent and subconfluent TM4SF5-expessing cells, and certain piled-up cells were in the middle of cell division. Whereas TM4SF5-null cells showed a controlled growth, TM4SF5-expressing cells grew continuously, progressed to S-phase even under confluent conditions, and formed more foci in soft agar and tumors in nude mice. Such an uncontrolled growth was blocked by suppression of TM4SF5 or $\mathrm{p} 27^{\mathrm{Kip} 1}$, RhoA activation, and importantly by E-cadherin reexpression in TM4SF5-expresing cells. That is, contact inhibition loss leading to uncontrolled growth and tumorigenesis was caused by TM4SF5-mediated cell-cell contact loss.

\section{Methods}

Cells. Pooled clones (control Cp and TM4SF5-expressing pooled Tp cell clones) and single cell-driven clones (TM4SF5-expressing T3, T7, T11, and T16) of hepatocarcinoma SNU449 (Korean Cell Bank) cells were prepared via infection with retrovirus for empty pLNCX or PLNCX with TM4SF5 (23). TM4SF5-expressing cells reconstituted with human E-cadherin (T3m and $\mathrm{T} 16 \mathrm{~m}$ ) were prepared from stable transfection of SNU449T3 and SNU449T16 cells with human E-cadherin cDNA (a gift from Barry M. Gumbiner, University of Virginia, Charlottesville, Virginia, USA).

Cells with or without endogenous TM4SF5. Huh7 cells stably transfected with shRNA against TM4SF5 (shTM4SF5, see below) were prepared by G418 ( $500 \mu \mathrm{g} / \mathrm{ml}$; A.G. Scientific Inc.) selection. Parental and stable cells were maintained in RPMI-1640/10\% FBS $/ 0.25 \mu \mathrm{g} / \mathrm{ml}$ gentamycin (Calbiochem) without or with G418 $(200 \mu \mathrm{g} / \mathrm{ml})$, respectively, at $37^{\circ} \mathrm{C}$ and $5 \% \mathrm{CO}_{2}$. Huh7, HepG2, and SNU368 hepatocytes and SNU601 gastric carcinoma cells endogenously expressing TM4SF5 and SNU449 and SNU398 hepatocytes expressing no TM4SF5 (Korean Cell Bank) were maintained in either DMEM-H (Huh7 and HepG2) or RPMI-1640 media (WelGENE Inc.) with 10\% FBS.

Cell lysate preparation and Western blots. Whole-cell lysates from cells under diverse experimental conditions were prepared as described previously (40). In certain experimental conditions, cells were transfected with indicated expression vectors, control or TM4SF5 shRNA (shTM4SF5; ref. 23), or infected with adenovirus for siRNA against $\mathrm{p} 27^{\text {Kip } 1}$ or scrambled sequence for the indicated periods (41). pEGFP-FLAG-GRAF and p190RhoGAP plasmids were kind gifts from J.M. Taylor and Keith Burridge, respectively (University of North Carolina, Chapel Hill, North Carolina, USA). In certain experimental conditions, cells were treated with $10 \mu \mathrm{M}$ cycloheximide (Sigma-Aldrich) for the indicated periods. Protein amounts of the lysates were normalized and then used in standard Western blots using antibodies. Antibodies for Western blots included anti-phospho-Y577FAK (BioSource International Inc.), -phospho- $\mathrm{Y}^{416} \mathrm{Src},-\mathrm{c}-\mathrm{Src}$, -RhoA, -p120 ctn, - $\beta$-catenin, - $\mathrm{pS}^{10} \mathrm{p}^{2} 7^{\mathrm{Kip} 1}{ }^{1}$, -Snail1 (Santa Cruz Biotechnology Inc.), -FAK, -E-cadherin, -ZO-1, -Rac1, -p190RhoGAP, -p27Kip1 (BD Biosciences), -GRAF, -hKIS (ABGENT), -desmoplakin (Serotec), $-\alpha$-tubulin, $-\alpha$-catenin, - $\alpha$-SMA (Sigma-Aldrich), or -TM4SF5 (prepared in our laboratory).
Anti-TM4SF5. A peptide covering the region of the second extracellular loop of TM4SF5 (amino acid residues from 120 to 132; NM-003963) conjugated with KLH was immunized to rabbits, and after boostings, sera were collected to check the anti-TM4SF5 immunoactivity via ELISA (A \& Pep Inc.) and immunoblots using TM4SF5 recombinant proteins and cell extracts. The sera were further purified by affinity chromatography using protein A/G-sepharose (Invitrogen).

Immunofluorescence microscopy. Cells were replated on 10\% FBS/RPMI1640-precoated coverslips and incubated overnight at $37^{\circ} \mathrm{C}$ to reach typical adhesion and spreading. Expression plasmid for FLAG-p27 $7^{\text {Kip } 1}$ was a kind gift from Naoya Fujita (University of Tokyo, Tokyo, Japan). Expressio plasmid for active mDia was a kind gift from S. Narumiya (Kyoto University, Kyoto, Japan). In certain experimental conditions, cells on coverslips were transfected or infected with diverse genetic reagents, as above, for the indicated periods. Alternatively, cells were treated with $10 \mathrm{mM}$ LPA (LC Laboratories) for 1 hour. Immunofluorescence staining or actin staining were processed as described previously (23). Antibodies for immunofluorescence included anti-E-cadherin, $-\beta$-catenin (Santa Cruz Biotechnology Inc.), -hKIS, -FLAG (ABGENT), -BrdU (Calbiochem), $-\mathrm{p} 27^{\text {Kip1 }}$ (BD Biosciences), and -ZO-1 (Zymed). Mounted samples were visualized by a fluorescence microscope (BX51; Olympus) or a confocal microscope (LSM 5 PASCAL; Carl Zeiss).

Immunoprecipitation. Coimmunoprecipitation between FAK and p190RhoGAP or GRAF was performed using whole-cell lysates from subconfluent SNU449Cp and SNU449Tp cells, as explained previously (40).

RhoA activity assay. RhoA activity was measured as described previously (23). RT-PCR. RT-PCR for $\mathrm{p} 27^{\mathrm{Kip} 1}$ was performed as described previously (42). The reactions used a sense ( $5^{\prime}$-TAACCCGGGACTTGGAGAAG- $\left.3^{\prime}\right)$ and an antisense ( $5^{\prime}$-GCTTCTTGGGCGTCTGCTC- $\left.3^{\prime}\right)$ primer, as shown in a previous study (43).

BrdU incorporation and cell growth analysis. BrdU incorporation analysis of confluent cells under different experimental conditions and cell growth analysis by viable cell counting were performed as described previously (44).

Soft agar assay. Cells without or with TM4SF5 expression were processed for soft agar assay as described previously (45). The plates were then incubated for 27 days in a $5 \% \mathrm{CO}_{2}$ incubator at $37^{\circ} \mathrm{C}$, with replenishment of medium containing $200 \mu \mathrm{g} / \mathrm{ml} \mathrm{G} 418$ and $10 \%$ FBS every other day. Colonies were imaged using a digital camera-equipped microscope.

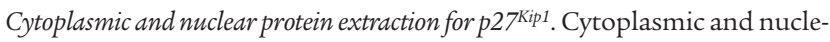
ar fractionation for $\mathrm{p} 27^{\mathrm{Kip} 1}$ levels in TM4SF5-expressing SNU449Tp and SNU449T16 cells were performed as described previously (34).

Mouse and tumor xenografts. Four- or 5-week-old female nude (BALB/ cAnNCrjBgi-nu) mice were purchased from Orient Co. Ltd. Mice were housed in a specific pathogen-free room under controlled temperature and humidity. All animal procedures were performed in accordance with the procedures in the Seoul National University Laboratory Animal Maintenance Manual and with Institutional Review Board approval. Mice age 5-6 weeks were injected subcutaneously in the flank with $1 \times 10^{7}$ viable SNU449, SNU449Cp, SNU449Tp, SNU449T16, SNU449T3m, or SNU449T16m cells. Tumor volumes were measured with a caliper and calculated using the following formula: volume $=\left(a \times b^{2}\right) / 2$, where $a$ is width at the widest point of the tumor and $b$ is maximal width perpendicular to $a$.

Human liver tissue sample. Normal and tumor liver tissues were obtained from patients in the Kyungpook National University Hospital (Daegu, Republic of Korea) from March to April 2007 with informed consent and with Institutional Review Board approval. Clinical and biological information was available for the tissue samples.

Tumor histology or Western blot analysis. Tumors were removed 6 weeks after injection of either SNU449Cp or SNU449T16 cells, fixed with formalin, and embedded in paraffin. Tumor masses were frozen immediately after 
surgery using liquid $\mathrm{N}_{2}$, prior to homogenization for cell extracts in $0.1 \%$ SDS containing RIPA buffer to use in Western blots. Alternatively, serial sections ( $6 \mu \mathrm{m}$ thick) cut from each paraffin block were stained with $\mathrm{H} \& \mathrm{E}$ or for $\mathrm{p} 27^{\text {Kip } 1}$ (1:50; BD Biosciences) or protein A/G affinity-purified antiTM4SF5 polyclonal serum (prepared in our laboratory; 1:100), prior to visualization using a microscope (BX51; Olympus).

Statistics. For comparisons of mean values, 1-tailed Student's $t$ tests were performed to determine whether the difference was significant. $P \leq 0.05$ was considered significant.

\section{Acknowledgments}

We authors thank Rudy L. Juliano (University of North Carolina at Chapel Hill, Chapel Hill, North Carolina, USA) for his valuable discussion on the manuscript. This work was supported by Korea
Science and Engineering Foundation (KOSEF) grants KOSEF-F012006-000-10018-0, KOSEF-2007-03536, and R11-2007-007-01004-0 funded by the Korean government (MOST) (to J.W. Lee).

Received for publication August 29, 2007, and accepted in revised form January 23, 2008.

Address correspondence to: Jung Weon Lee, Cancer Research Institute, Departments of Molecular and Clinical Oncology and Tumor Biology, College of Medicine, Seoul National University, 103, Daehangno, Jongno-gu, Seoul 110-799, Republic of Korea. Phone: 82-2-3668-7030; Fax: 82-2-766-4487; E-mail: jwl@snu.ac.kr.

Sin-Ae Lee and Sung-Yul Lee contributed equally to this work.
1. Thiery, J.P. 2002. Epithelial-mesenchymal transitions in tumour progression. Nat. Rev. Cancer. 2:442-454.

2. Hirohashi, S., and Kanai, Y. 2003. Cell adhesion system and human cancer morphogenesis. Cancer Sci. 94:575-581.

3. Stipp, C.S., Kolesnikova, T.V., and Hemler, M.E. 2003. Functional domains in tetraspanin proteins. Trends. Biochem. Sci. 28:106-112.

4. Berditchevski, F. 2001. Complexes of tetraspanins with integrins: more than meets the eye. J. Cell Sci. 114:4143-4151.

5. Charrin, S., et al. 2003. Multiple levels of interactions within the tetraspanin web. Biochem. Biophys. Res. Commun. 304:107-112.

6. Yang, X., et al. 2004. Palmitoylation supports assembly and function of integrin-tetraspanin complexes. J. Cell Biol. 167:1231-1240.

7. Hynes, R.O. 2002. Integrins: bidirectional, allosteric signaling machines. Cell. 110:673-687.

8. Brakebusch, C., and Fassler, R. 2003. The integrinactin connection, an eternal love affair. EMBO J. 22:2324-2333.

9. Juliano, R.L., et al. 2004. Integrin regulation of cell signalling and motility. Biochem. Soc. Trans. 32:443-446.

10. Yamada, K.M., and Even-Ram, S. 2002. Integrin regulation of growth factor receptors. Nat. Cell Biol. 4:E75-E76.

11. Lee, J.W., and Juliano, R. 2004. Mitogenic signal transduction by integrin- and growth factor receptor-mediated pathways. Mol. Cells. 17:188-202.

12. Hall, A. 2005. Rho GTPases and the control of cell behaviour. Biochem. Soc. Trans. 33:891-895.

13. Schmitz, A.A., Govek, E.E., Bottner, B., and Van Aelst, L. 2000. Rho GTPases: signaling, migration, and invasion. Exp. Cell Res. 261:1-12.

14. Besson, A., Gurian-West, M., Schmidt, A., Hall, A., and Roberts, J.M. 2004. p27 ${ }^{\text {Kip } 1}$ modulates cell migration through the regulation of RhoA activation. Genes Dev. 18:862-876.

15. Boehm, M., et al. 2002. A growth factor-dependent nuclear kinase phosphorylates $\mathrm{P} 27^{\mathrm{Kip} 1}$ and regulates cell cycle progression. EMBOJ. 21:3390-3401.

16. Sato, S., Fujita, N., and Tsuruo, T. 2002. Regulation of kinase activity of 3-phosphoinositide-dependent protein kinase- 1 by binding to 14-3-3. J. Biol. Chem. 277:39360-39367.

17. Ciaparrone, M., et al. 1998. Localization and expression of $\mathrm{p} 27^{\mathrm{KIP} 1}$ in multistage colorectal carcinogenesis. Cancer Res. 58:114-122.

18. Singh, S.P., et al. 1998. Loss or altered subcellular localization of p27 in Barrett's associated adeno- carcinoma. Cancer Res. 58:1730-1735.

19. Baldassarre, G., et al. 1999. Overexpressed cyclin D3 contributes to retaining the growth inhibitor p27 in the cytoplasm of thyroid tumor cells. J. Clin. Invest. 104:865-874.

20. Wright, M.D., Ni, J., and Rudy, G.B. 2000. The L6 membrane proteins - a new four-transmembrane superfamily. Protein Sci. 9:1594-1600.

21. Muller-Pillasch, F., et al. 1998. Identification of a new tumour-associated antigen TM4SF5 and its expression in human cancer. Gene. 208:25-30.

22. Pascual-Le Tallec, L., Dulmet, E., Bertagna, X., and de Keyzer, Y. 2002. Identification of genes associated with the corticotroph phenotype in bronchial carcinoid tumors. J. Clin. Endocrinol. Metab. 87:5015-5022.

23. Lee, S.Y., et al. 2006. Focal adhesion and actin organization by a cross-talk of TM4SF5 with integrin $\alpha 2$ are regulated by serum treatment. Exp. Cell Res. 312:2983-2999.

24. Clark, E.A., King, W.G., Brugge, J.S., Symons, M., and Hynes, R.O. 1998. Integrin-mediated signals regulated by members of the Rho family of GTPases. J. Cell Biol. 142:573-586.

25. Mitra, S.K., Hanson, D.A., and Schlaepfer, D.D. 2005. Focal adhesion kinase: in command and control of cell motility. Nat. Rev. Mol. Cell Biol. 6:56-68.

26. Rodier, G., et al. 2001. p27 cytoplasmic localization is regulated by phosphorylation on Ser10 and is not a prerequisite for its proteolysis. $E M B O J$. 20:6672-6682.

27. Besson, A., Assoian, R.K., and Roberts, J.M. 2004. Regulation of the cytoskeleton: an oncogenic function for CDK inhibitors? Nat. Rev. Cancer. 4:948-955.

28. Besson, A., et al. 2006. A pathway in quiescent cells that controls $\mathrm{p}^{27^{\mathrm{Kip}} 1}$ stability, subcellular localization, and tumor suppression. Genes Dev. 20:47-64.

29. Coqueret, O. 2003. New roles for p21 and p 27 cellcycle inhibitors: a function for each cell compartment? Trends Cell Biol. 13:65-70.

30. Oh, M.-A., et al. 2007. PKC $\delta$ and cofilin activation affects peripheral actin reorganization and cell-cell contact in cells expressing integrin $\alpha 5$ but not its tailless mutant. J. Cell Sci. 120:2717-2730.

31. Sherr, C.J., and Roberts, J.M. 1999. CDK inhibitors: positive and negative regulators of G1-phase progression. Genes Dev. 13:1501-1512.

32. Fujita, N., Sato, S., Katayama, K., and Tsuruo, T. 2002. Akt-dependent phosphorylation of $\mathrm{p}^{2} 7^{\mathrm{Kip} 1}$ promotes binding to 14-3-3 and cytoplasmic localization. J. Biol. Chem. 277:28706-28713.

33. Sekimoto, T., Fukumoto, M., and Yoneda, Y. 2004.
14-3-3 suppresses the nuclear localization of threonine 157-phosphorylated p27 Kip1. EMBO J. 23:1934-1942.

34. Liang, J., et al. 2002. PKB/Akt phosphorylates p27, impairs nuclear import of p27 and opposes p27mediated G1 arrest. Nat. Med. 8:1153-1160.

35. Bhowmick, N.A., et al. 2001. Transforming growth factor- $\beta 1$ mediates epithelial to mesenchymal transdifferentiation through a RhoA-dependent mechanism. Mol. Biol. Cell. 12:27-36.

36. Charrasse, S., et al. 2002. N-cadherin-dependent cell-cell contact regulates Rho GTPases and $\beta$-catenin localization in mouse $\mathrm{C} 2 \mathrm{C} 12$ myoblasts. J. Cell Biol. 158:953-965.

37. Hirohashi, S. 1998. Inactivation of the E-cadherinmediated cell adhesion system in human cancers. Am. J. Pathol. 153:333-339.

38. Gottardi, C.J., Wong, E., and Gumbiner, B.M. 2001. E-cadherin suppresses cellular transformation by inhibiting $\beta$-catenin signaling in an adhesion-independent manner. J. Cell Biol. 153:1049-1060.

39. Pirone, D.M., et al. 2006. An inhibitory role for FAK in regulating proliferation: a link between limited adhesion and RhoA-ROCK signaling. J. Cell Biol. 174:277-288.

40. Lee, M.-S., et al. 2005. The signaling network of transforming growth factor $\beta 1$, protein kinase $\mathrm{C} \delta$, and integrin underlies the spreading and invasiveness of gastric carcinoma cells. Mol. Cell. Biol. 25:6921-6936.

41. Tamamori-Adachi, M., et al. 2004. Down-regulation of $\mathrm{p}^{27^{\mathrm{Kip}} 1}$ promotes cell proliferation of rat neonatal cardiomyocytes induced by nuclear expression of cyclin D1 and CDK4: evidence for impaired Skp2-dependent degradation of p27 in terminal differentiation. J. Biol. Chem. 279:50429-50436.

42. Kim, Y.-B., et al. 2005. Cell adhesion status-dependent histone acetylation is regulated through intracellular contractility-related signaling activities. J. Biol. Chem. 280:28357-28364.

43. Wang, C., et al. 2005. Activation of $\mathrm{p}^{27^{\text {Kip } 1}}$ Expression by E2F1. A negative feedback mechanism. J. Biol. Chem. 280:12339-12343.

44. Kim, H.-P., et al. 2005 . TGF- $\beta 1$-mediated activations of c-Src and Rac1 modulate levels of cyclins

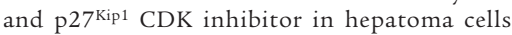
replated on fibronectin. Biochim. Biophys. Acta. 1743:151-161.

45. Kim, Y.-B., Lee, S.-Y., Ye, S.-K., and Lee, J.W. 2007. Epigenetic regulation of integrin-linked kinase expression depending on adhesion of gastric carcinoma cells. Am. J. Physiol. Cell Physiol. 292:C857-C866. 